\title{
Symmetry and stationary points of a free energy: The case of superfluid ${ }^{3} \mathrm{He}$
}

\author{
Christoph Bruder* \\ Physik-Department, Technische Universität München, D-8046 Garching, Federal Republic of Germany \\ Dieter Vollhardt \\ Max-Planck-Institut für Physik und Astrophysik, Werner-Heisenberg-Institut für Physik, Postfach 4012 12, \\ D-8000 München 40, Federal Republic of Germany
}

(Received 27 January 1986)

\begin{abstract}
The symmetry of the free energy of superfluid ${ }^{3} \mathrm{He}$ is used to investigate stationary points which correspond to possible superfluid phases. For this purpose a complete classification of both continuous and discrete subgroups of the symmetry of the free-energy functional describing $p$-wave superfluidity of ${ }^{3} \mathrm{He}, G=\mathrm{SO}(3) \times \mathrm{SO}(3) \times \mathrm{U}(1)$, is presented. The corresponding order parameters of phases with broken symmetry are determined explicitly. It is shown that all superfluid phases previously found in the literature by minimizing the Ginzburg-Landau functional are included in this classification. Hence, in all these phases the symmetry $G$ is only partly broken such that they still contain a residual symmetry. This is true both for inert and noninert states. The concept of broken relative symmetries - of great importance for an understanding of the properties of superfluid ${ }^{3} \mathrm{He}$-appears as a very natural feature in such a group-theoretical treatment. The classification is also applied to superfluid ${ }^{3} \mathrm{He}$ in a magnetic field and with dipolar coupling. Using the methods of differential topology developed by Michel, we show that the symmetry properties of phases and their energy are related. This is explicitly verified by applying the classification to two systems whose free-energy functional is simpler than that of superfluid ${ }^{3} \mathrm{He}$ (e.g., $d$-wave pairing or superfluidity in neutron stars) and whose minima are known from analytic calculation. Hence, grouptheoretical methods prove to be a very valuable tool for investigating the stationary points of complicated free-energy functionals in condensed-matter physics, in particular of those where an analytical minimization seems untractable as in superfluid ${ }^{3} \mathrm{He}$.
\end{abstract}

\section{INTRODUCTION}

The superfluid phases of ${ }^{3} \mathrm{He}$ are unusually complex states of condensed matter which exhibit a unique wealth of physical properties. ${ }^{1-6}$ This fact is in striking contrast to the extreme simplicity of the system on the microscopic level, since ${ }^{3} \mathrm{He}$ is composed of spherical, i.e., structureless, atoms. Its properties result from the high density of the atoms, their strong interaction and, in particular, the fermionic character of the ${ }^{3} \mathrm{He}$ atoms due to the nuclear spin $I=\frac{1}{2}$.

The superfluid phases known today are characterized by a condensation of Cooper pairs into a state with relative angular momentum $l=1$ and, consequently, total spin $S=1$ (spin triplet, $p$-wave pairing). This implies an anisotropic pair wave function and, generally, leads to anisotropic properties of the system. The associated order parameter is then given by a complex $3 \times 3$ matrix. ${ }^{1-4,6}$ The unusually large number of internal degrees of freedom associated with this tensor order parameter are responsible for the many different properties of superfluid ${ }^{3} \mathrm{He}$. Indeed, no other single physical system known so far shows as many physical phenomena as do these superfluid phases, which incorporate the behavior of liquid crystals, magnetically ordered states, and superfluids.

Phase transitions are usually connected with the breaking of a symmetry. ${ }^{7}$ This concept has proven to be extremely fruitful in many areas in physics besides condensed-matter physics, e.g., in high-energy physics, ${ }^{8}$ where the different families of particles are interpreted as the result of symmetry breaking on a cosmic level.

In most of the systems of condensed-matter physics with a broken symmetry (superconductors, superfluid ${ }^{4} \mathrm{He}$, ferromagnet) the order parameter has a simple structure and therefore the corresponding energy functional is also simple. This functional can then easily be treated analytically to find its minima which correspond to possible phases with broken symmetry. Unfortunately, for superfluid ${ }^{3} \mathrm{He}$ this has not yet been possible in full generality. ${ }^{9-15}$ In view of the difficulty of the problem which involves 18 variational parameters it remains doubtful whether this is mathematically possible at all.

The search for possible phases can proceed along quite different lines. For example, it may ask for the solution of the idealized weak-coupling problem as done by Balian and Werthamer, ${ }^{9}$ Ambegaokar and Mermin ${ }^{10}$ and Mermin and Stare, ${ }^{11}$ or for the solution under certain constraints for the order parameter (like a unitarity assumption ${ }^{11}$ ), or by demanding certain magnetic properties of resulting phases $^{12}$ known from the experiment. A different line of approach is due to Barton and Moore ${ }^{13,14}$ who concentrate on those phases whose order parameters do not depend on the parameters $\beta_{i}$, i.e., the prefactors of the fourth-order terms in the free-energy functionals. These phases are called "inert"- their order parameters do not change their structure upon a change of external parameters; only the 
overall magnitude of the gap will change. However, while Barton and Moore ${ }^{13}$ find all possible inert states, they also point out that "noninert" states cannot be neglected in a full analysis of the minimization problem. Later, Jones ${ }^{15}$ studied the problem using a combination of algebraic and geometric methods and thus provided the most complete analysis up to date. He derived a general equation for extrema of the free energy (which still cannot be completely solved), and was able to show that some of the inert phases found previously ${ }^{13}$ were not minima but only saddle points of the free energy. Furthermore he discussed the connection between the values of the $\beta_{i}$ and the existence of noninert states. ${ }^{15}$

Considering the states found in the above-mentioned works one may ask for the stable states which, on physical grounds, are most likely to be formed. In this case the $\beta_{i}$ parameters necessary for stability should probably not be too far away from their weak-coupling values. ${ }^{1,2,12}$ It appears as if this problem had largely been solved. On the other hand, one may ask the more general question regarding minima of the free energy for arbitrary $\beta_{i}$. In this case one has to concentrate on noninert states. Indeed, they cannot be neglected because, as will be shown below, they are the phases describing ${ }^{3} \mathrm{He}-A$ and $-B$ in the presence of an external magnetic field.

In view of the formidable-and as yet unsolvedproblems connected with the search for minima of the free-energy functional we suggest proceeding in a completely different way. It is based on the following observation: We find that all phases which correspond to minima of the free energy and which have so far been discussed in the literature are described by a respective order parameter with a remaining internal symmetry; that is, although these phases appear as states with a broken symmetry relative to the underlying energy functional, the symmetry is not totally broken, but only partly. Hence, we observe that an order parameter with a residual internal symmetry corresponds to a phase with a lower energy than one where all symmetries are broken. ${ }^{16}$ To our knowledge there exists no general proof that this has to be so. On the other hand, using somewhat simpler order parameters than that of superfluid ${ }^{3} \mathrm{He}$, we will give explicit examples where this observation can be shown to be rigorously true.

The connection between the symmetry of an order parameter and its energy with respect to a free energy has been studied within the context of high-energy physics, but also within a more mathematical context. For example, Jaric ${ }^{17}$ shows that, given finite groups and a realvalued order parameter, the minima of a GinzburgLandau functional must always have a residual symmetry. Using geometrical methods $\mathrm{Kim}^{18}$ described a way for determining the energy and residual symmetry of the Higgs potential minima. This method was then applied to a quartic Higgs potential with SU(5) symmetry. ${ }^{19}$ The results were used to discuss the hierarchical symmetry breaking of SU(5) grand unification theory. Abud and Sartori $^{20}$ prove that within a Ginzburg-Landau theory with algebraically independent fourth-order terms, the minima correspond to states with a remaining symmetry. Clearly, these methods are also relevant for problems in condensed-matter physics. For example, Jaric ${ }^{21}$ shows how a symmetry classification may be used to describe a ferroelectric phase transition in perovskites. Similarly, $\mathrm{Kim}^{22}$ illustrates the method by applying it to a Ginzburg-Landau potential describing $\mathrm{BaTiO}_{3}$ in order to explain the three successive phase transitions known to occur in this system. Clearly, there is also a close connection between the symmetry of a phase and the shape (i.e., "smoothness") of its order parameter: In general, the higher the symmetry, the smoother the order parameter. This point has been addressed by Anderson and Brinkman $^{2}$ in connection with superfluid ${ }^{3} \mathrm{He}$.

In view of these facts the main aim of this paper is to find all those order-parameter structures which still possess some of the original symmetry of the underlying free-energy functional. Using group-theoretical methods, we will show that there is a systematic way to derive these order parameters, namely, by finding all subgroups $H$ of this underlying symmetry-group $G$. Concerning continuous subgroups, this question has first been addressed by Golo and Monastyrskiy ${ }^{23}$ and, in particular, by Mineev ${ }^{24}$ (based on the unpublished work of Volovik). In this way Mineev ${ }^{24}$ obtained all inert states which can be obtained by a factorization using continuous groups. As will be shown below there are other inert phases which cannot be found in this way but only by using discrete symmetries. Studying the possibility of $f$-wave pairing in superfluid ${ }^{3} \mathrm{He}$, Barton and Moore ${ }^{14}$ tried to employ finite groups to find inert states.

It is important to note that a symmetry classification is independent of any specific form of an energy functional. In particular, one is not restricted to a Ginzburg-Landau expansion which is only valid in the very vicinity of the transition. All we need to know is the symmetry of the free-energy functional. Of course, the states found by the symmetry classification will not necessarily be minima of the free energy. On the other hand, once they have been found, any particular energy functional will be able to determine which one of them is lowest in energy.

The concept described above will be helpful in all those cases where an analytic minimization of a free-energy function proves to be an intractable problem (i.e., usually, whenever the order parameter has a very complicated structure).

Similar symmetry classifications as described abovebut with quite a different aim-have recently been shown to be very useful for studying heavy-fermion superconductivity ${ }^{25-28}$ and rotating ${ }^{3} \mathrm{He}$ and ${ }^{4} \mathrm{He}$ (Ref. 29). In both cases a symmetry-group factorization is performed in order to derive the respective free-energy functionals.

The paper is organized as follows. In Sec. II we discuss the structure of the ${ }^{3} \mathrm{He}$ order parameter and the related free-energy functional. Simpler examples than superfluid ${ }^{3} \mathrm{He}$, e.g., the usual superconductor and the ferromagnet are used to clarify the necessary concepts. In Sec. III a short review of the group-theoretical methods needed in the investigation of continuous subgroups is presented. In Sec. IV we will derive all continuous subgroups of the underlying symmetry group and will explicitly calculate the corresponding order parameters. The investigations of continuous groups in Sec. IV turn out to be incomplete in- 
sofar as some of the phases found by Barton and Moore ${ }^{13}$ are missing. We find them to be invariant under certain discrete transformations. This shows that discrete symmetries have to be included in our treatment. Accordingly a complete classification of discrete subgroups of $G$ is presented in Sec. V. The states thus found in Secs. IV and $V$ are not necessarily stationary points of the free energy. However, in some cases it is possible to give sufficient conditions for them to be extrema. Using methods from differential topology, we are able to give a new interpretation of inert states; this is done in Sec. VI. In Sec. VII we will consider the restrictions imposed on the underlying symmetry due to an external magnetic field or the microscopic spin-orbit coupling. The appropriate classification of states is presented. In Sec. VIII two models of systems described by a tensor order parameter similar to superfluid ${ }^{3} \mathrm{He}$, whose respective Ginzburg-Landau functional can be minimized analytically, will be discussed (one of them is a ${ }^{3} P_{2}$ superfluid thought to be realized in neutron stars). We will show that all minima of the free energy can be found by symmetry considerations alone. A conclusion follows in Sec. IX.

\section{ORDER PARAMETER AND GINZBURG-LANDAU THEORY}

In contrast to superfluid ${ }^{3} \mathrm{He}$ the order parameters of most other systems in condensed-matter physics (with the exception of liquid crystals) are quite simple. Correspondingly the free-energy functional describing the energy change relative to the system with unbroken symmetry is also simple.

(1) In a conventional superconductor the order parameter is represented by a single complex parameter, $\Delta=\Delta_{0} e^{i \phi}=\Delta_{1}+i \Delta_{2}$, where $\Delta_{0}$ is the amplitude and $\phi$ is the phase ("gauge"). Close to $T_{c}$ the free-energy functional involving this order parameter is given by

$$
F=\alpha \Delta \Delta^{*}+\beta\left(\Delta \Delta^{*}\right)^{2} \text {. }
$$

It is invariant under an arbitrary change of the phase $e^{i \phi} \rightarrow e^{i \phi^{\prime}}$, i.e., under a gauge transformation, equivalent to a $U(1)$ symmetry. In the superconducting state a particular value of $\phi$ is spontaneously preferred such that the $\mathrm{U}(1)$ symmetry is broken. Clearly, (2.1) can trivially be minimized; it has a single minimum given by $|\Delta|^{2}=-\alpha / 2 \beta$.

(2) In a ferromagnet the order parameter is the magnetization $\overrightarrow{\mathrm{S}}$, a real, three-component vector. The free-energy functional is given by

$$
F=\alpha \overrightarrow{\mathbf{S}} \cdot \overrightarrow{\mathbf{S}}+\boldsymbol{\beta}(\overrightarrow{\mathbf{S}} \cdot \overrightarrow{\mathbf{S}})^{2},
$$

which also only contains a single fourth-order term. It is invariant under arbitrary three-dimensional rotations of the spin, i.e., under an $S O(3)$ symmetry. In the ferromagnetic state, however, a particular direction of $\overrightarrow{\mathbf{S}}$ is spontaneously chosen, such that the system is now only invariant under rotations around $\overrightarrow{\mathrm{S}}$ itself: The $\mathrm{SO}(3)$ symmetry is reduced to a residual $U(1)$ symmetry. As in the first example, (2.2) has a single minimum at $|\overrightarrow{\mathbf{S}}|^{2}=-\alpha / 2 \beta$.

(3) In superfluid ${ }^{3} \mathrm{He}$, a $p$-wave, spin-triplet condensate
( $l=1, S=1$ ) is formed. ${ }^{1-6}$ The BCS pair wave function is thus given by

$$
\Delta(\hat{\mathbf{k}})=\sqrt{3} i \Delta(T) A_{\mu j} \hat{k}_{j}\left(\tau^{\mu} \cdot \tau^{2}\right)
$$

i.e., by a symmetric matrix in spin space describing $S=1$ and a linear $\hat{\mathbf{k}}$ relation describing $l=1$. (Here, $\tau^{i}$ are the Pauli spin matrices.) The normalized order parameter $A_{\mu j}$ is hence a quantity with two indices: one in spin space and one in orbit space. It can be written as a complex $3 \times 3$ matrix containing 18 real parameters. If we only consider the interactions responsible for the formation of the condensed state, i.e., neglect small, residual interactions like the dipole interaction, the free energy must be invariant under separate three-dimensional rotations in real (or orbit) space, in spin space, and under a gauge transformation. The symmetry group allowing for all these symmetries and which is least restrictive in the energy, is then given by ${ }^{1}$

$$
G=[\mathrm{SO}(3)]_{\overrightarrow{\mathrm{S}}} \times[\mathrm{SO}(3)]_{\overrightarrow{\mathrm{L}}} \times[\mathrm{U}(1)]_{\phi} .
$$

Here the indices $\vec{S}, \overrightarrow{\mathrm{L}}, \phi$ indicate spin space, orbit space, and gauge, respectively. The free-energy functional in the Ginzburg-Landau regime can then be obtained by contracting all indices in the general second- and fourth-order terms in a way compatible with (2.4). The result is ${ }^{11}$

$$
\begin{aligned}
F=\alpha \Delta^{2} \operatorname{Tr}\left(\mathbf{A} \mathbf{A}^{\dagger}\right)+\frac{1}{2} \Delta^{4}\{ & \beta_{1}|\operatorname{Tr}(\mathbf{A} \tilde{\mathbf{A}})|^{2}+\beta_{2}\left[\operatorname{Tr}\left(\mathbf{A} \mathbf{A}^{\dagger}\right)\right]^{2} \\
& +\beta_{3} \operatorname{Tr}\left[(\mathbf{A} \tilde{\mathbf{A}})(\mathbf{A} \tilde{\mathbf{A}})^{*}\right] \\
& +\beta_{4} \operatorname{Tr}\left[\left(\mathbf{A} \mathbf{A}^{\dagger}\right)^{2}\right] \\
& \left.+\beta_{5} \operatorname{Tr}\left[\left(\mathbf{A} \mathbf{A}^{\dagger}\right)\left(\mathbf{A} \mathbf{A}^{\dagger}\right)^{*}\right]\right\}
\end{aligned}
$$

These are now five fourth-order terms. The coefficients $\alpha$ and $\beta_{i}$ depend on external parameters like pressure and temperature. They can easily be calculated within weakcoupling theory ${ }^{11}$ where they obey the relation $\beta_{2}=\beta_{3}=\beta_{4}=-\beta_{5}=-2 \beta_{1}$. On the other hand, an explicit calculation of strong-coupling corrections is difficult. $^{30-32}$ Concerning the symmetry considerations addressed in this paper the actual values of $\alpha$ and $\beta_{i}$ are not important; we consider them to be as given. The superfluid phases which may be found near $T_{c}$ are given by the minima of (2.5). The absolute minimum corresponds to the stable equilibrium phase, local minima only yield metastable states.

While in the first two examples the free energy can be trivially minimized, this has not yet been possible in full generality in the case of (2.5) describing superfluid ${ }^{3} \mathrm{He}$ close to $T_{c}$. In order to support our observation, explained in Sec. I, that minima of the free energy generally possess a residual internal symmetry (and hence can be found by symmetry considerations), two more examples of systems described by a tensor order parameter and their associated free energies will be presented in Sec. VIII. They are close in complexity to $(2.5)$ but can still be minimized analytically. The results will be compared with those found by the respective symmetry classification of possible states. In these cases it will turn out that the symmetry classification yields all minima. 


\section{GROUP THEORY}

From a group-theoretical point of view, "broken symmetry" means that below the transition the lowest state of the system is no longer invariant under the full group $G$ of the symmetry transformations but only under a subgroup $H \subset G$. Therefore $H$ describes the remaining (residual) symmetry of the state. The group $G$ may be factorized with respect to $H$ :

$$
G=R \times H .
$$

Here the coset-space $R=G / H$ contains all transformations that change the ground state of the system. Hence $R$ describes the broken symmetries of the system.

In the case of the transition from a paramagnetic to a ferromagnetic state the respective groups are $G=\mathrm{SO}(3)$ and $H=\mathrm{SO}(2)[=\mathrm{U}(1)]$. In the paramagnetic state the system is invariant under three-dimensional rotations in space $[\mathrm{SO}(3)]$, while in the ferromagnetic states only rotations around the spontaneous magnetization $\overrightarrow{\mathbf{S}}$, described by $U(1)$, leave the ground state invariant. Therefore the broken symmetries, corresponding to any rotation changing the direction of $\overrightarrow{\mathrm{S}}$, are given by $R=\mathrm{SO}(3) / \mathrm{U}(1)=S^{2}$, the rotations represented by the two-sphere.

In superfluid ${ }^{3} \mathrm{He}$ the symmetry-group $G$ is given by (2.4). At this point we are interested in the various factorizations of $G$ with respect to continuous subgroups $H$ under which the order parameter $A_{\mu j}$ is still invariant. Once we have found these groups the specific structure of $A_{\mu j}$ can easily be determined. The factorization with respect to groups leading to inert states has first been discussed by Mineev $^{24}$ (based on unpublished work of Volovik).

Every group element $h \in H$ corresponds to a transformation leaving $A_{\mu j}$ invariant:

$$
h A_{\mu j}=A_{\mu j} \text {. }
$$

It may be written as

$$
\begin{aligned}
h & =\exp (i \mathbf{T}) \\
& \simeq 1+i \mathbf{T},
\end{aligned}
$$

where $\mathbf{T}$ are the generators of the infinitesimal transformations of the group $H$, i.e.,

$$
\mathbf{T A}=0 \text {. }
$$

The order-parameter $\mathrm{A} \equiv A_{\mu j}$ has the transformation properties of a "bivector:" it transforms like a vector both with respect to its spin part (first index) and to its orbital part (second index). This fact, combined with the invariance properties of the free energy under the group of transformations $(2.4)$ concerning rotations in spin and orbit space and a gauge transformation, imply that $T$ can be written as a linear combination of these transformations:

$$
\mathbf{T}=\overrightarrow{\mathbf{a}} \cdot \overrightarrow{\mathbf{L}}+\overrightarrow{\mathrm{b}} \cdot \overrightarrow{\mathbf{S}}+c \boldsymbol{\Phi}
$$

Here $\overrightarrow{\mathbf{S}}=\left(\mathbf{S}^{x}, \mathbf{S}^{y}, \mathbf{S}^{z}\right)$ and $\overrightarrow{\mathbf{L}}=\left(\mathbf{L}^{x}, \mathbf{L}^{y}, \mathbf{L}^{z}\right)$ are the threecomponent spin operator and orbital angular momentum operator, respectively (i.e., $3 \times 3$ matrices). They are given by

$$
\begin{aligned}
& \left(\mathbf{L}^{i}\right)_{j k}=-i \hbar \epsilon_{i j k}, \\
& \left(\mathbf{S}^{\mu}\right)_{\nu \lambda}=-i \hbar \epsilon_{\mu \nu \lambda} .
\end{aligned}
$$

Furthermore, $\boldsymbol{\Phi}=-i \hbar \partial / \partial \phi$ is the operator for a gauge transformation, with

$$
\boldsymbol{\Phi} \mathbf{A}=\mathbf{A} \text {. }
$$

While $\overrightarrow{\mathbf{S}}$ only acts on the first index of $A_{\mu j}, \overrightarrow{\mathbf{L}}$ only acts on the second one and $\Phi$ only operates on the overall phase.

There are altogether seven generators $(\overrightarrow{\mathbf{L}}, \overrightarrow{\mathbf{S}}, \boldsymbol{\Phi})$-a fact which is also reflected in the existence of seven infinitesimal, but otherwise arbitrary, real parameters $\overrightarrow{\mathrm{a}}=\left(a_{x}, a_{y}, a_{z}\right), \overrightarrow{\mathrm{b}}=\left(b_{x}, b_{y}, b_{z}\right), c$. The relations (3.7) and (3.8) define Lie algebras among the $L^{i}, S^{\mu}$ components

$$
\begin{aligned}
& {\left[\mathbf{L}^{i}, \mathbf{L}^{j}\right]=i \hbar \epsilon_{i j k} \mathbf{L}^{k},} \\
& {\left[\mathbf{S}^{\mu}, \mathbf{S}^{\nu}\right]=i \hbar \epsilon_{\mu v \lambda} \mathbf{S}^{\lambda} .}
\end{aligned}
$$

Inserting (3.6) $-(3.9)$ into (3.5) leads to

$$
a_{i} \epsilon_{i j k} A_{\mu k}+b_{\lambda} \epsilon_{\lambda \mu v} A_{v j}+i c A_{\mu j}=0
$$

which is a set of nine homogeneous equations for the nine complex components of the order parameter $A_{\mu j}$ in terms of the parameters $a_{i}, b_{\mu}, c(i, \mu=1,2,3)$. By constructing all possible linear combinations of the generators in (3.6), i.e., by finding all continuous subgroups $H \neq 1$ of $G$, we are able to derive all those order-parameter structures that still possess some of the original symmetry (2.4).

\section{CONTINUOUS SYMMETRIES}

We will now list all possible linear combinations of the infinitesimal generators, i.e., all possible continuous subgroups $H$ of $G$. Equation (3.11) is then used to calculate the components of $A_{\mu j}$. The respective investigations including discrete symmetries, e.g., rotations by $2 \pi / m$ in spin and orbit space accompanied by discrete changes of the overall phase, will be treated in the next section. It should be stressed that the order parameters thus obtained are only determined up to a rotation in spin and orbit space and an overall phase factor, because there is no fixed coordinate system.

First we start with the case where the phase invariance is separately broken [i.e., $c=0$ in (3.6)].

(1) $c=0$ and $a=b$. This implies

$$
\mathbf{T}=\overrightarrow{\mathbf{a}} \cdot(\overrightarrow{\mathbf{L}}+\overrightarrow{\mathbf{S}})
$$

corresponding to $H=[\mathrm{SO}(3)]_{\overrightarrow{\mathrm{L}}+\overrightarrow{\mathrm{S}}}$. Here, the order parameter is still invariant under joint, three-dimensional rotations of $\overrightarrow{\mathrm{L}}$ and $\overrightarrow{\mathrm{S}}$. In this sense it is the most symmetric of all possible order parameters. Solving (3.11) one finds

$$
A_{\mu j}=\frac{1}{\sqrt{3}} \delta_{\mu j}
$$

which is a special case of the more general form (see the remarks made above)

$$
A_{\mu j}=\frac{1}{\sqrt{3}} e^{i \phi} R_{\mu j}(\hat{\mathbf{n}}, \theta),
$$


where $\boldsymbol{R}_{\boldsymbol{\mu}}$ is a rotation matrix describing relative rotations of spin and orbit space, represented by a rotation axis $\hat{\mathbf{n}}$ and a rotation angle $\theta$. Note, that this more general state is invariant under simultaneous rotations described by a matrix $O_{l j}$ in orbit space and $\left(\mathbf{R O R}{ }^{-1}\right)_{\nu \mu}$ in spin space. This is, of course, the Balian-Werthamer (BW) state. The explicit phase factor $e^{i \phi}$ is due to the separately broken gauge invariance. To determine the degeneracy space $R=G / H$, i.e., the broken symmetries, we factorize $G$ with respect to $H$ :

$$
\begin{aligned}
R & =\left\{[\mathrm{SO}(3)]_{\overrightarrow{\mathrm{s}}} \times[\mathrm{SO}(3)]_{\overrightarrow{\mathrm{L}}} \times[\mathrm{U}(1)]_{\phi}\right\} /[\mathrm{SO}(3)]_{\overrightarrow{\mathrm{L}}+\overrightarrow{\mathrm{S}}} \\
& =[\mathrm{SO}(3)]_{\overrightarrow{\mathrm{L}}, \overrightarrow{\mathrm{s}}} \times[\mathrm{U}(1)]_{\phi} .
\end{aligned}
$$

We observe that the factorization of $G$ with respect to $[\mathrm{SO}(3)]_{\overrightarrow{\mathbf{L}}}+\overrightarrow{\mathrm{S}}$, the group of all joint rotations of $\overrightarrow{\mathrm{L}}$ and $\overrightarrow{\mathrm{S}}$, leaves us with $[\mathrm{U}(1)]_{\phi}$ as well as $[\mathrm{SO}(3)]_{\vec{L}, \overrightarrow{\mathbf{s}}}$-the group of all relative rotations of $\overrightarrow{\mathrm{L}}$ and $\overrightarrow{\mathrm{S}}$. Hence, in the $\mathrm{BW}$ state the relative spin-orbit symmetry as well as the gauge symmetry is broken. This broken symmetry is called "spontaneously broken spin-orbit symmetry" (SBSOS). The term was introduced by Leggett ${ }^{33}$ who was the first to realize the existence of this particular symmetry breaking.

As elucidated by Liu and Cross ${ }^{34}$ the new content of SBSOS lies in the breaking of a relative symmetry. The symmetries of rotations both in spin and orbit space are here also broken, but not independently-actually a linear combination of these symmetries is broken. It implies that while the constituent degrees of freedom in spin and orbit space are not long-range ordered their relative orientation is.

A system with a broken relative symmetry consequently behaves like one in which the symmetries of the constituent degrees of freedom are individually broken but which cannot distinguish between them-it confuses them. ${ }^{35}$ We see already here that the notion of a broken relative symmetry appears quite naturally within a group-theoretical formulation. It occurs every time when a product of groups is not simply factorized by factorizing each individual group.

With the exception of the BW phase having $H=\mathrm{SO}(3)$, the symmetry-subgroup $H$ is composed of $\mathrm{U}(1)$ subgroups only (i.e., we can choose $a_{x}=a_{y}=b_{x}=b_{y}=0$ ). The set of equations (3.11) determining the nine complex parameters $A_{\mu j}$ is then given by

$a_{z}\left(\delta_{j 1} A_{\mu 2}-\delta_{j 2} A_{\mu 1}\right)+b_{z}\left(\delta_{\mu 1} A_{2 j}-\delta_{\mu 2} A_{1 j}\right)+i c A_{\mu j}=0$.

One can show that it separates into four independent subsystems, belonging to different sets of $A_{\mu j}$ components. For $A_{\mu j}$ to be nonzero, the determinant $D$ of the matrix (4.6) has to vanish:

$$
D=D_{1} D_{2} D_{3} D_{4}
$$

Here, the $D_{i}$ are the determinants of the submatrices belonging to the $\boldsymbol{A}_{\mu j}$ components as indicated:

$$
\begin{aligned}
& D_{1}=\left[\left(a_{z}-b_{z}\right)^{2}-c^{2}\right]\left[\left(a_{z}+b_{z}\right)^{2}-c^{2}\right] \\
& D_{2}=b_{z}^{2}-c^{2} \quad\left(A_{11}, A_{12}, A_{21}, A_{22}\right), \\
& D_{3}=a_{z}^{2}-c^{2}\left(A_{31}, A_{32}\right), \\
& D_{4}=i c \quad\left(A_{33}\right) .
\end{aligned}
$$

This way of writing allows for a simple and systematic investigation of all linear combinations of $a_{z}, b_{z}$, and $c$ in (4.6). Depending on which of the $D_{i}$ we choose to be zero (nonzero) we can immediately determine which of the $\boldsymbol{A}_{\mu j}$ components are nonzero (zero). To construct a linear combination of infinitesimal generators means that we impose conditions on them ("break" the symmetry). One can easily convince oneself that in the case of (4.6) a single condition (say, $c=0$ ) implies a remaining symmetry $H=\mathrm{U}(1) \times \mathrm{U}(1)$, leading to an inert phase. On the other hand, imposing more than one condition (say, $c=0$ and $a_{z}=b_{z}$ ) implies an even more restricted symmetry, $H=\mathrm{U}(1)$, leading to noninert phases. We now complete the list of possible phases.

(2) $c=0 ; a_{2}, b_{2}$ arbitrary. This implies

$$
\mathbf{T}=a_{z} \mathbf{L}^{z}+b_{z} \mathbf{S}^{z} \text {, }
$$

and hence $H=[\mathrm{U}(1)]_{S_{z}} \times[\mathrm{U}(1)]_{L_{z}}$. Since $D_{1}, D_{2}, D_{3} \neq 0$ and $D_{4}=0$, one immediately obtains the polar state

$$
A_{\mu j}=\left(\begin{array}{lll}
0 & 0 & 0 \\
0 & 0 & 0 \\
0 & 0 & 1
\end{array}\right) \text {. }
$$

Its order parameter is still invariant under separate rotations around the preferred directions in spin and orbit space which are both real unit vectors. Accordingly, its broken symmetries are given by

$$
\begin{aligned}
R= & \left\{[\mathrm{SO}(3)]_{\overrightarrow{\mathrm{s}}} /[\mathrm{U}(1)]_{S_{z}}\right\} \times\left\{[\mathrm{SO}(3)]_{\overrightarrow{\mathrm{L}}} /[\mathrm{U}(1)]_{L_{z}}\right\} \\
& \times[\mathrm{U}(1)]_{\phi} \\
= & \left(S^{2}\right)_{\overrightarrow{\mathrm{s}}} \times\left(S^{2}\right)_{\overrightarrow{\mathrm{L}}} \times[\mathrm{U}(1)]_{\phi}
\end{aligned}
$$

i.e., besides the phase invariance, also the invariance with respect to rotations in spin and orbit space other than around the preferred directions themselves, is broken. The polar state is unique in that it is the only phase without a broken relative symmetry.

(3) $c=0 ; b_{z}=0, a_{z}$ arbitrary. This implies

$$
\mathbf{T}=a_{z} \mathbf{L}^{z},
$$

and hence $H=[\mathrm{U}(1)]_{L_{z}}$. Since $D_{1}, D_{3} \neq 0$ and $D_{2}, D_{4}=0$, the order parameter has the structure

$$
A_{\mu j}=p\left(\begin{array}{lll}
0 & 0 & A \\
0 & 0 & B \\
0 & 0 & C
\end{array}\right)
$$

with $p=\left[|A|^{2}+|B|^{2}+|C|^{2}\right]^{-1 / 2}$ and $A, B, C$ complex. The condition $b_{z}=0$ implies that in this noninert state the symmetry with respect to rotations in spin space has been completely broken: 


$$
\begin{aligned}
R & =[\mathrm{SO}(3)]_{\overrightarrow{\mathrm{S}}} \times\left\{[\mathrm{SO}(3)]_{\overrightarrow{\mathrm{L}}} /[\mathrm{U}(1)]_{L_{z}}\right\} \times[\mathrm{U}(1)]_{\phi} \\
& =[\mathrm{SO}(3)]_{\overrightarrow{\mathrm{S}}} \times\left(\mathrm{S}^{2}\right)_{\overrightarrow{\mathrm{L}}} \times[\mathrm{U}(1)]_{\phi},
\end{aligned}
$$

insofar as it is less symmetric (i.e., more restrictive) than the polar state (4.10) which is seen to be a special case of (4.14), with $A=B=0$.

(4) $c=0 ; a_{z}=0, b_{z}$ arbitrary. This implies

$$
\mathbf{T}=b_{z} \mathbf{S}^{2} \text {, }
$$

and hence $H=[\mathrm{U}(1)]_{S_{z}}$. It corresponds to the previous state with orbital and spin components interchanged; i.e.,

$$
A_{\mu j}=p\left(\begin{array}{lll}
0 & 0 & 0 \\
0 & 0 & 0 \\
A & B & C
\end{array}\right),
$$

and $R=\left(S^{2}\right)_{\overrightarrow{\mathrm{s}}} \times[\mathrm{SO}(3)]_{\overrightarrow{\mathrm{L}}} \times[\mathrm{U}(1)]_{\phi}$. Here the rotation symmetry in orbital space is completely broken. Again the polar state is obtained for $A=B=0$.

(5) $c=0$ and $a_{z} \mp b_{z}=0$. This implies

$$
\mathbf{T}=a_{z}\left(\mathbf{L}^{\mathbf{z}} \pm \mathbf{S}^{\mathbf{z}}\right) \text {, }
$$

and hence $H=[U(1)]_{L_{z}+S_{z}}$. Since $D_{1}=D_{4}=0$ and $D_{2}, D_{3} \neq 0$, this leads to

$$
A_{\mu j}=p\left(\begin{array}{ccc}
A & B & 0 \\
\pm B & \mp A & 0 \\
0 & 0 & C
\end{array}\right)
$$

where $A, B, C$ are complex numbers and $p=\left[2\left(|A|^{2}+|B|^{2}\right)+|C|^{2}\right]^{-1 / 2}$. This state is a generalized representation of the so-called $\zeta$ phase found by Barton and Moore. ${ }^{13}$ However, the name "oblate state" appears to be more useful, because it actually describes a squashed spherical state. In fact, (4.20) represents precisely the order-parameter structure of the $B_{2}$ phase, ${ }^{4}$ i.e., of the $B$ phase in an external magnetic field [with $\left.A=\frac{1}{2} i\left(\Delta_{\uparrow \uparrow}+\Delta_{\downarrow \downarrow}\right), B=-\frac{1}{2} i\left(\Delta_{\uparrow \uparrow}-\Delta_{\downarrow \downarrow}\right), C=\Delta_{\uparrow \downarrow}\right]$. Furthermore, by flattening the sphere completely, i.e., on choosing $B=C=0$, one obtains the usual planar state. Thus the actual planar state contains both continuous and discrete symmetries. The broken symmetries of (4.20) are found to be

$$
\begin{aligned}
R & =\left(\left\{[\mathrm{SO}(3)]_{\overrightarrow{\mathrm{S}}} \times[\mathrm{SO}(3)]_{\overrightarrow{\mathrm{L}}}\right\} /[\mathrm{U}(1)]_{S_{z}+L_{z}}\right) \times[\mathrm{U}(1)]_{\phi} \\
& =\left(S^{2}\right)_{\overrightarrow{\mathrm{S}}} \times\left(S^{2}\right)_{\overrightarrow{\mathrm{L}}} \times[\mathrm{U}(1)]_{S_{z}, L_{z}} \times[\mathrm{U}(1)]_{\phi}
\end{aligned}
$$

which includes the broken relative symmetry $[\mathrm{U}(1)]_{S_{z}, L_{z}}$ with respect to rotations in spin and orbit space.

(6) $a_{z}+c=0, b_{z}$ arbitrary. This implies

$$
\mathbf{T}=a_{z}\left(\mathbf{L}^{\mathbf{z}}-\boldsymbol{\Phi}\right)+b_{\mathbf{z}} \mathbf{S}^{\mathbf{z}},
$$

and hence $H=[\mathrm{U}(1)]_{L_{z}-\phi} \times[\mathrm{U}(1)]_{S_{z}}$. Since $D_{3}=0$ and $D_{1}, D_{2}, D_{4} \neq 0$, one finds

$$
A_{\mu j}=\frac{1}{\sqrt{2}}\left(\begin{array}{lll}
0 & 0 & 0 \\
0 & 0 & 0 \\
1 & i & 0
\end{array}\right),
$$

which is, of course, the axial state. It is still invariant under rotations around one direction (the $z$ direction) in spin space and under a joint transformation of the phase and a rotation around $L_{z}$. The broken symmetry is obtained as

$$
\begin{aligned}
R= & \left\{[\mathrm{SO}(3)]_{\overrightarrow{\mathrm{S}}} /[\mathrm{U}(1)]_{S_{z}}\right\} \\
& \times\left\{[\mathrm{SO}(3)]_{\overrightarrow{\mathrm{L}}} \times[\mathrm{U}(1)]_{\phi} /[\mathrm{U}(1)]_{L_{z}-\phi}\right\} \\
= & \left(S^{2}\right)_{\overrightarrow{\mathrm{S}}} \times\left(S^{2}\right)_{\overrightarrow{\mathrm{L}}} \times[\mathrm{U}(1)]_{L_{z}, \phi},
\end{aligned}
$$

i.e., the invariance with respect to rotations of directions in spin and orbit space, as well as the relative gauge-orbit symmetry $[\mathrm{U}(1)]_{L_{z}}$ is broken. ${ }^{36}$

(7) $a_{z}+c=0 ; b_{z}=0$. This implies

$$
\mathbf{T}=a_{z}\left(\mathbf{L}^{z}-\Phi\right) \text {, }
$$

and hence $H=[\mathrm{U}(1)]_{L_{z}-\phi}$. Since $D_{1}=D_{3}=0$ and $D_{2}, D_{4} \neq 0$, one finds

$$
A_{\mu j}=\frac{p}{\sqrt{2}}\left(\begin{array}{lll}
A & i A & 0 \\
B & i B & 0 \\
C & i C & 0
\end{array}\right),
$$

where $p=\left[|A|^{2}+|B|^{2}+|C|^{2}\right]^{-1 / 2}$ and $A, B, C$ are complex numbers. In this state spin-rotation symmetry is completely broken $(\vec{b}=0)$ :

$$
\begin{aligned}
R & =\left\{[\mathrm{SO}(3)]_{\overrightarrow{\mathrm{s}}} \times[\mathrm{SO}(3)]_{\overrightarrow{\mathrm{L}}} \times[\mathrm{U}(1)]_{\phi}\right\} /[\mathrm{U}(1)]_{L_{z}-\phi} \\
& =[\mathrm{SO}(3)]_{\overrightarrow{\mathrm{S}}} \times\left(S^{2}\right)_{\overrightarrow{\mathrm{L}}} \times[\mathrm{U}(1)]_{L_{z}, \phi}
\end{aligned}
$$

Because of the more restrictive symmetry requirements its order parameter is less symmetric than both that of the $A$ phase $(A=B=0)$ and that of the $A_{1}$ phase $(B=-i A$, $C=0$ ). In fact it is a general representation of the $A_{2}$ phase, which is obtained by choosing $A=\frac{1}{2}\left(\Delta_{\uparrow \uparrow}+\Delta_{\downarrow \downarrow}\right)$, $B=\frac{1}{2} i\left(\Delta_{\uparrow \uparrow}-\Delta_{\downarrow \downarrow}\right), C=0$. Note, that the orientation of the spin-coordinate system in the present case $\left(\hat{\mathbf{d}} \equiv \hat{\mathbf{d}}_{x}, \hat{\mathbf{e}} \equiv \hat{\mathbf{d}}_{y}\right)$ has been chosen differently from the one for the $A$ phase $\left(\hat{\mathbf{d}} \equiv \hat{\mathbf{d}}_{z}\right)$, which is, however, of no importance.

(8) $b_{z}+c=0 ; a_{z}=0$. This implies

$$
\mathbf{T}=b_{\mathbf{z}}\left(\mathbf{S}^{\mathbf{z}}-\mathbf{\Phi}\right) \text {, }
$$

and hence $H=[\mathrm{U}(1)]_{S_{z}-\phi}$. It can be obtained from the previous state by interchanging orbital and spin space, i.e.,

$$
A_{\mu j}=\frac{p}{\sqrt{2}}\left(\begin{array}{ccc}
A & B & C \\
i A & i B & i C \\
0 & 0 & 0
\end{array}\right) .
$$

Here rotation symmetry in orbital space is completely broken ( $\overrightarrow{\mathrm{a}}=0)$, i.e., $R=[\mathrm{SO}(3)]_{\overrightarrow{\mathrm{L}}} \times\left(S^{2}\right)_{\overrightarrow{\mathrm{S}}} \times[\mathrm{U}(1)]_{S_{z}, \phi}$.

(9) $b_{z}+c=0, a_{z}$ arbitrary. This implies

$$
\mathbf{T}=a_{\mathbf{z}} \mathbf{L}^{z}+b_{z}\left(\mathbf{S}^{z}-\Phi\right),
$$

and hence $H=[\mathrm{U}(1)]_{L_{z}} \times[\mathrm{U}(1)]_{S_{z}-\phi}$. Since $D_{2}=0$ and 
$D_{1}, D_{3}, D_{4} \neq 0$, one finds

$$
A_{\mu j}=\frac{1}{\sqrt{2}}\left(\begin{array}{lll}
0 & 0 & 1 \\
0 & 0 & i \\
0 & 0 & 0
\end{array}\right),
$$

which is called " $\beta$ phase" (Ref. 13). Its symmetry properties, etc., are obtained by interchanging the orbital and the spin parts of the Anderson-Brinkman-Morel (ABM) state (4.24) $(A \rightarrow \widetilde{A})$. Accordingly, in this case a relative gauge-spin symmetry is broken.

(10) $c=a_{z}-b_{z}$. This implies

$$
\mathbf{T}=a_{z}\left(\mathbf{L}^{z}+\Phi\right)+b_{z}\left(\mathbf{S}^{z}-\Phi\right),
$$

and hence $H=[\mathrm{U}(1)]_{L_{z}+\phi} \times[\mathrm{U}(1)]_{S_{z}-\phi}$. Here one has $D_{1}=0$ and $D_{2}, D_{3}, D_{4} \neq 0$, such that

$$
A_{\mu j}=\frac{1}{2}\left(\begin{array}{ccc}
1 & i & 0 \\
-i & 1 & 0 \\
0 & 0 & 0
\end{array}\right) \text {, }
$$

which is the representation of the $A_{1}$ phase. The broken symmetry is given by

$$
\begin{aligned}
R & =\left(S^{2}\right)_{\overrightarrow{\mathrm{s}}} \times\left(S^{2}\right)_{\overrightarrow{\mathrm{L}}} \times\left(\left\{[\mathrm{U}(1)]_{S_{z}} \times[\mathrm{U}(1)]_{L_{z}} \times[\mathrm{U}(1)]_{\phi}\right\} /\left\{[\mathrm{U}(1)]_{L_{z}+\phi} \times[\mathrm{U}(1)]_{S_{z}-\phi}\right\}\right) \\
& =\left(S^{2}\right)_{\overrightarrow{\mathrm{s}}} \times\left(S^{2}\right)_{\overrightarrow{\mathrm{L}}} \times[\mathrm{U}(1)]_{L_{z}, S_{z}, \phi} .
\end{aligned}
$$

In this case a $\mathrm{U}(1)$ symmetry involving a linear combination of all three symmetries, i.e., a gauge-spin-orbit symmetry, is broken as first discussed by Liu. ${ }^{37}$

(11) $a_{z}+c=0$ and $b_{z}+c=0$. This implies

$$
\mathbf{T}=a_{2}\left(\mathbf{L}^{2}+\mathbf{S}^{z}-\Phi\right),
$$

and hence $H=[\mathrm{U}(1)]_{L_{2}+S_{z}-\phi}$. Here one has $D_{1}, D_{4} \neq 0$ and $D_{2}=D_{3}=0$ such that

$$
A_{\mu j}=p\left(\begin{array}{ccc}
0 & 0 & A \\
0 & 0 & i A \\
B & i B & 0
\end{array}\right)
$$

with $p=\left[2\left(|A|^{2}+|B|^{2}\right)\right]^{-1 / 2}$, which has been called the " $\epsilon$ phase" (Ref. 13); one may view it as a linear combination of the $A$ and the $\beta$ phase. This noninert state is only invariant under joint $U(1)$ rotations of spin, gauge, and orbital variables. In a way it is complementary to the $A_{1}$ phase. Its broken symmetries are

$$
\begin{aligned}
R & =\left(S^{2}\right)_{\overrightarrow{\mathrm{s}}} \times\left(S^{2}\right)_{\overrightarrow{\mathrm{L}}} \times\left(\left\{[\mathrm{U}(1)]_{S_{z}} \times[\mathrm{U}(1)]_{L_{z}} \times[\mathrm{U}(1)]_{\phi}\right\} /[\mathrm{U}(1)]_{L_{z}+S_{z}-\phi}\right) \\
& =\left(S^{2}\right)_{\overrightarrow{\mathrm{s}}} \times\left(S^{2}\right)_{\overrightarrow{\mathrm{L}}} \times[\mathrm{U}(1)]_{S_{z}, \phi} \times[\mathrm{U}(1)]_{L_{z}, \phi},
\end{aligned}
$$

i.e., a relative spin-gauge and a relative gauge-orbit symmetry is broken.

(12) $c=a_{z}+b_{z}$ and $c+b_{z}=0$. This implies

$$
\mathbf{T}=c\left(2 \mathbf{L}^{z}-\mathbf{S}^{z}+\Phi\right)
$$

and hence $H=[\mathrm{U}(1)]_{2 L_{\mathrm{z}}-S_{\mathrm{z}}+\phi}$. We are dealing here with a different linear combination of infinitesimal generators than in case (11), and hence with a different symmetry $H$. Since $D_{1}=D_{2}=0$ and $D_{3}, D_{4} \neq 0$, one has

$$
A_{\mu j}=p\left(\begin{array}{ccc}
A & -i A & B \\
-i A & -A & i B \\
0 & 0 & 0
\end{array}\right)
$$

with $p=\left[2\left(2|A|^{2}+|B|^{2}\right)\right]^{-1 / 2}$. This noninert state is a superposition of the $A_{1}$ and the $\beta$ phase. As in the case of the $\epsilon$ phase it has two separate broken relative $\mathrm{U}(1)$ symmetries.

(13) $c=a_{z}+b_{z}$ and $c+a_{z}=0$. This implies

$$
\mathbf{T}=-c\left(\mathbf{L}^{z}-2 \mathbf{S}^{z}-\Phi\right),
$$

and hence $H=[\mathrm{U}(1)]_{L_{z}-2 S_{z}-\phi}$. It is obtained from (12) by interchanging the spin and orbital parts of the order- parameter $(A \rightarrow \tilde{A})$.

The last two states are similar to case (11) because the symmetry-group $\boldsymbol{H}$ involves particular linear combinations of all three symmetries, i.e., particular joint rotations. Consequently, their broken relative symmetries will all be very similar.

We have thus found 13 order-parameter structures which still contain the symmetry of a continuous subgroup $H$ of $G$ : one with a remaining $\mathrm{SO}(3)$ symmetry, four with a $U(1) \times U(1)$ symmetry (they all lead to inert states), and eight with a remaining $U(1)$ symmetry, yielding noninert states. The states (3), (4), (7), (8), (12), and (13) have not yet been discussed in the literature.

In Fig. 1 the consecutive factorization of the symmetry-group $G$ is shown schematically. An arrow running diagonally always indicates that the factorization involves more than one group, such that the resulting phase has a broken relative symmetry.

We note, that the phase with the highest remaining symmetry [SO(3)] is the $B$ phase. It is known to be stable, i.e., to have the lowest energy, in the largest part of the experimentally determined phase diagram. Its order parameter is the smoothest of all phases. ${ }^{2}$ Next are the inert 


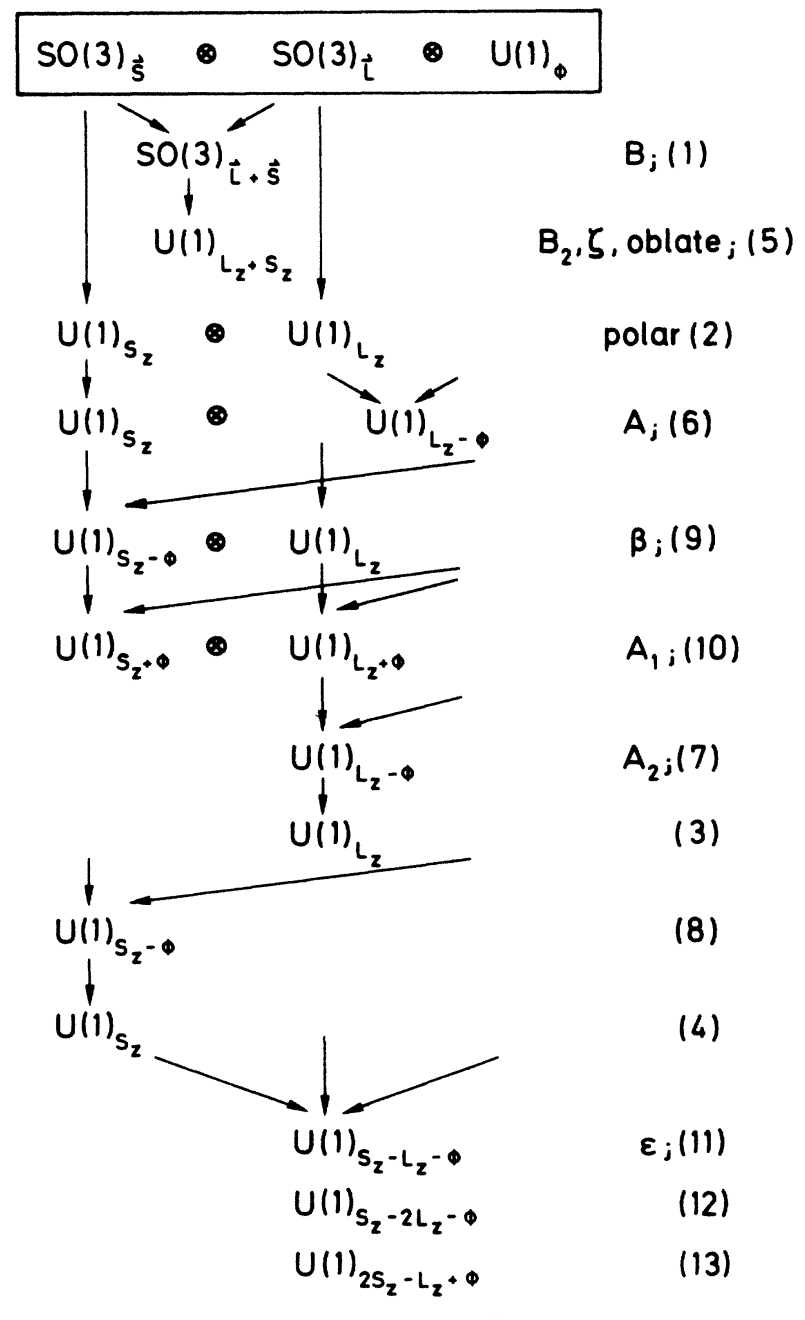

FIG. 1. The symmetry classification of the group $G=[\mathrm{SO}(3)]_{\vec{S}} \times[S O(3)]_{\overrightarrow{\mathrm{L}}} \times[\mathrm{U}(1)]_{\phi}$ into continuous subgroups is shown schematically. Diagonal arrows indicating a factorization that involves more than one group at a time, lead to broken relative symmetries. On the right-hand side the names of the phases (if any) and their respective numbers in the listing of Sec. IV are indicated.

states with a $\mathrm{U}(1) \times \mathrm{U}(1)$ symmetry, two of which are the $A$ and $A_{1}$ phases which are also found to be stable in a certain region of the phase diagram.

\section{DISCRETE SYMMETRIES}

The investigation of the continuous subgroups of $G=[\mathrm{SO}(3)]_{\overrightarrow{\mathrm{S}}} \times[\mathrm{SO}(3)]_{\overrightarrow{\mathrm{L}}} \times[\mathrm{U}(1)]_{\phi}$ in Sec. IV, yielded 13 different order-parameter structures. Seven of them $(1,2,5,6,9,10,11)$ have already been discussed in the literature, ${ }^{11-15}$ i.e., have been found to be stationary points (or even minima) of the free energy functional (2.5). ${ }^{11-13,15}$ This shows that the structure of these phases is given by symmetry alone: the order-parameter matrix $A_{\mu j}$ is determined by the requirement to be invariant under the action of the respective continuous subgroup of $G$.

On the other hand, there are four more phases which are known to be stationary points of the free energy: the $\alpha$ phase, ${ }^{13}$ the bipolar phase, and the axiplanar phase ${ }^{12}$ (as well as the $\delta$ phase, whose order parameter is the transpose of that of the axiplanar phase ${ }^{13}$ ). They have not been found by the analysis of Sec. IV involving continuous symmetries. If we adhere to the observation that the minima of the free energy correspond to phases whose order parameter has a residual internal symmetry, we have to search for symmetries other than continuous ones, i.e., discrete symmetries. Therefore, our aim in this section will be to classify all discrete subgroups of $G$ and to determine the order-parameter states invariant under their action.

The continuous subgroups of $G$ can be specified by the infinitesimal generators of $G$ : Each element is written as an exponential of a linear combination of generators. There is an analogue in the theory of discrete groups: A discrete group is generated by a subset of elements, i.e., each element can be written as a product of powers of generators. The cyclic groups $C_{n}$, for example (i.e., the rotations through $2 \pi k / n, k=1,2, \ldots, n$ ), are generated by one element only. However, because of the large number of different discrete subgroups of $G$ these generators turn out not to be so useful. We will therefore resort to another approach.

Let $H_{D}$ be a discrete subgroup of $G$ (2.4). Each element of $G$ can be written as a triple $\left(\mathbf{r}^{5}, \mathbf{r}^{0}, \exp \left(i \phi_{0}\right)\right)$ describing a rotation $\boldsymbol{r}^{s}$ in spin space, a rotation $r^{0}$ in orbit space, and a gauge transformation $\exp \left(i \phi_{0}\right)$. Clearly, $\boldsymbol{r}^{s}$ and $r^{\circ}$ will have to be elements of discrete subgroups of $S O(3)$, and $\exp \left(i \phi_{0}\right)$ an element of a discrete subgroup of $\mathrm{U}(1)$.

The discrete subgroups of $\mathrm{SO}(3)$ and $\mathrm{U}(1)$ are well known. For $\mathrm{SO}(3)$ they are given by ${ }^{38}$

(i) $C_{n}$ : the point group of rotations about the $z$ axis through $2 \pi k / n$, with $k=1,2, \ldots, n$. The elements of $C_{n}$ are denoted by $c_{n}^{k}$.

(ii) $D_{n}$ : generated by the point group $C_{n}$ and an additional rotation through $\pi$ around an orthogonal axis.

(iii) $T, O, Y$ : the point groups of the tetrahedron, the cube, and the icosahedron, respectively.

The subgroups of $\mathrm{U}(1)$ are given by $C_{n}$.

For each discrete subgroup $H_{D}$ of $G$ there exists a minimal direct product subgroup $H_{s} \times H_{o} \times H_{\phi}$ of $G$ that contains $H_{D}$. "Minimal" means that there is no directproduct subgroup of $H_{s} \times H_{o} \times H_{\phi}$ that contains $H_{D}$. It is easy to construct $H_{s}, H_{o}$, and $H_{\phi}$ by projection of $H_{D}$ onto the factors $[\mathrm{SO}(3)]^{\text {spin }}$, [SO(3) $]^{\text {orbit }}$, and $\mathrm{U}(1)$ of $G$.

This construction may be applied to continuous subgroups as well. If, for example, we take the symmetry group of the $B$ phase, $[\mathrm{SO}(3)]_{\overrightarrow{\mathrm{s}}+\overrightarrow{\mathrm{L}}}$, the trivial result is that $\mathrm{SO}(3) \times \mathrm{SO}(3)$ is the minimal direct product containing $[\mathrm{SO}(3)]_{\overrightarrow{\mathrm{S}}+\overrightarrow{\mathrm{L}}}$.

We will see later that $H_{\phi}$, the discrete group of gauge transformations, is essentially determined once $H_{s}$ and $H_{o}$ are given. It is therefore sufficient to elucidate the structure of the spin and orbital parts of $H_{D}$ with respect to $H_{s} \times H_{o}$.

The discrete subgroups $H_{D}$ of $H_{s} \times H_{o}$ can be though of as follows. There exist invariant subgroups $H_{s}^{\prime}$ of $H_{s}$ 
and $H_{o}^{\prime}$ of $H_{o}$ such that the factor groups $H_{s} / H_{s}^{\prime}$ and $H_{o} / H_{o}^{\prime}$ are isomorphic (i.e., one-to-one). (A subgroup $H$ of $G$ is called invariant if $g \mathrm{Hg}^{-1}=H$ for all $g \in G$. Invariant subgroups are necessary for the construction of factor groups.) The group $H_{D}$ is then given by the set of all pairs $\left(h_{s}, h_{o}\right)$ with $h_{s} \in H_{s} / H_{s}^{\prime}$ and $h_{o} \in H_{o} / H_{o}^{\prime}$ where $h_{s}$ and $h_{o}$ are isomorphic elements.

Following Toledano et al. ${ }^{39}$ we use the notation $H_{D}=\left(H_{s} / H_{s}^{\prime} ; H_{o} / H_{o}^{\prime}\right)$. A proof that all subgroups $H_{D}$ are of this type has been given by Bruder. ${ }^{40}$ Since $H_{s}$ and $H_{o}$ were chosen such that $H_{s} \times H_{o}$ was the minimal direct product containing $H_{D}$, we do not consider nontrivial subgroups of $H_{s} \times H_{o}$ that are direct products.

Some examples will explain this construction.

(i) $H_{s}=C_{2}$ and $H_{o}=C_{3}$. The invariant subgroups are given by the trivial group $E$ and the groups themselves. Therefore the only possibility to obtain isomorphic factor groups is to take $H_{D}=\left(C_{2} / C_{2} ; C_{3} / C_{3}\right)=C_{2} \times C_{3}$.

(ii) $H_{s}=H_{o}=C_{3}$. The invariant subgroups are $E$ and $C_{3}$, hence either $H_{D}=\left(C_{3} / C_{3} ; C_{3} / C_{3}\right)=C_{3} \times C_{3}$ or $H_{D}=\left(C_{3} / E ; C_{3} / E\right)=\left[(e, e),\left(c_{3}^{1}, c_{3}^{1}\right),\left(c_{3}^{2}, c_{3}^{2}\right)\right]$.

(iii) $H_{s}=C_{4}$ and $H_{o}=C_{2}$. The first possibility is $H_{D}=C_{4} \times C_{2}$ as before. The group $C_{4}$ has a nontrivial invariant subgroup, namely, $C_{2}$. The factor group $C_{4} / C_{2}$ is isomorphic to $C_{2}$; hence we find

$$
\begin{aligned}
H_{D} & =\left(C_{4} / C_{2} ; C_{2}\right) \\
& =\left[\left(c_{2}^{0}, c_{4}^{0}\right),\left(c_{2}^{0}, c_{4}^{2}\right),\left(c_{2}^{1}, c_{4}^{1}\right),\left(c_{2}^{1}, c_{4}^{3}\right)\right] .
\end{aligned}
$$

Several notations of these groups exist in the literature [e.g., $\left.C_{4}\left(C_{2}\right)\right]^{26}$

Following this recipe, we can write down all discrete subgroups of $\mathrm{SO}(3) \times \mathrm{SO}(3)$ : The invariant subgroups of $C_{n}, D_{n}, T, O$, and $Y$ are known, and it is easy to calculate $H_{s} / H_{s}^{\prime}$ and $H_{o} / H_{o}^{\prime}$. Once all discrete subgroups of $\mathrm{SO}(3) \times \mathbf{S O}(3)$ are found, we can proceed to determine the order-parameter states invariant under their action together with the appropriate gauge transformations.

The states are obtained by solving systems of linear equations as in the case of continuous subgroups. If $\boldsymbol{A}_{\mu j}$ is to be invariant under the transformations of the discrete group

$$
H_{D}=\left[\left(\mathbf{r}_{k}^{s}, \mathbf{r}_{k}^{o}, \exp \left(i \phi_{k}\right)\right)\right],
$$

where $k$ is an index which we use to denote the elements of $H_{D}$, one has to solve

$$
\mathbf{r}_{k}^{s} \mathbf{A} \tilde{\mathbf{r}}_{k}^{o} \exp \left(i \phi_{k}\right)=\mathbf{A} \text { for all } k,
$$

i.e., one has to determine $A_{\mu j}$ and $\phi_{k}$ for given $r_{k}^{s}, r_{k}^{o} \in S O(3)$. These equations can be solved for arbitrary $\mathbf{r}_{k}^{s}, \mathbf{r}_{k}^{o}$ (Ref. 40). Equation (5.2) can be seen to break up into four systems of equations if the rotations are about the $z$ axis. The orientation of the rotation axes is not important, the solution is essentially determined by the rotation angles $\alpha_{k}$ and $\beta_{k}$ of $r_{k}^{s}$ and $r_{k}^{o}$. (Different orientations of rotation axes lead to different, but equivalent forms of the order parameter.) The solution of (5.2) is straightforward but tedious and will not be reproduced here. The most important general result is that

$$
\left|\varphi_{k}\right|=\left|\alpha_{k} \pm \beta_{k}\right| \text { or }\left|\alpha_{k}\right|,\left|\beta_{k}\right| \text { or } 0, \pi \text {. }
$$

This explains our earlier remark that the gauge group is determined by the spin and orbital parts of $H_{D}$. If, for example, $\alpha_{k}=\beta_{k}=\pi / 2, \varphi_{k}$ must take the values 0 or $\pi / 2$ or $\pi$ unless $A_{\mu j}=0$.

Having solved (5.2) for all possible discrete subgroups $H_{D}$ of $G$ (2.4), we can now give a complete classification of all superfluid order-parameter states that have a residual symmetry. The complete list shows a certain redundancy: Some of the states can be generated by several different groups.

The first result of the classification in Table I is that all the phases with a continuous symmetry are also produced by discrete symmetry requirements. This was to be expected since $C_{m}$ approximates $\mathrm{U}(1)$ for large $m$. It is surprising, however, that already small discrete groups can force the order parameter into a definite state. An example is given by the polar phase: it is invariant under $D_{\infty} \times D_{\infty}$, but is already generated by $D_{2} \times D_{2}$.

It is interesting to note, that some of the phases that appear in the classification of continuous symmetries are found to be invariant under additional discrete symmetry transformations. For example, the $A$ phase turns out to be invariant under $D_{n} \times C_{m}$ plus appropriate gauge transformations ( $n$ and $m$ are arbitrary which establishes an invariance under $D_{\infty} \times C_{\infty}$ plus gauge transformations). This is the additional $Z_{2}$ symmetry mentioned in Refs. 24 and 41.

Our motivation for investigating discrete symmetries was the fact that some of the inert phases are not invariant under the action of continuous groups. Table I proves that the bipolar, planar, and $\alpha$ phases, which were not found by continuous requirements contain a discrete symmetry:

(i) the bipolar phase is produced by $\left(D_{4} / C_{2} ; D_{4} / C_{2}\right)$;

(ii) the planar phase by $\left(D_{m} / E ; D_{m} / E\right)$ for $m>1$; and

(iii) the $\alpha$ phase by $(T / E ; T / E)$.

(The planar and $\alpha$ phases appear in a different form than that given in Ref. 13. Nonetheless they are equivalent up to a trivial rotation of the coordinate systems.) As a result we find that the classification of continuous and discrete subgroups of $\mathrm{SO}(3) \times \mathrm{SO}(3) \times \mathrm{U}(1)$ yields all inert phases.

It is interesting that these phases-previously determined by the zero points of systems of nonlinear equations - can be found by mere symmetry requirements. The symmetry classification is sometimes lengthy, but one does not encounter any substantial difficulties in carrying it through. In the next section we will look more closely at this connection between algebra and analysis.

The noninert phases found by Barton and Moore ${ }^{13}$ are obviously not determined by symmetry alone: Their structure depends on the coefficients in the free energy, and symmetry considerations cannot reproduce this dependence. Nevertheless even they appear because each of them is also invariant under certain transformations:

(i) the $\epsilon$ and $\zeta$ phases already appeared in Sec. IV; they are phases with a continuous symmetry;

(ii) the axiplanar and $\delta$ phase can be written in the form ${ }^{13}$ 
TABLE I. Order-parameter states invariant under the action of discrete subgroups of $G(2.4)$. In the first column the spin-orbit part of a symmetry group is shown. The second column presents a generating set of group elements, indicating the combination of gauge transformations with spin and orbital transformations. The notation is the same as before, e.g., $\left\{c_{n}^{1}, c_{k}^{1}, 2 \pi / l\right\}$ denotes a rotation through $2 \pi / n$ in spin space, $2 \pi / k$ in orbit space, and a multiplication by $\exp (2 \pi / l)$ (gauge transformation). Here, $c_{n}^{k}$ is a rotation about the $z$ axis, $c_{n, x}^{k}$ a rotation about the $x$ axis, $c_{n, x+y+z}^{k}$ a rotation about a diagonal of a cube. The identity element is denoted by $e$, the corresponding trivial group by $E$. The third column shows the order-parameter state determined by the symmetry group; the normalization factor has been neglected for simplicity. All parameters appearing in the matrices are arbitrary complex constants. The fourth column presents all other groups that lead to the same order-parameter states, e.g., subgroups of the group in the first column. In the cases where the gauge transformations have not been explicitly specified they are the same as in column 2 .

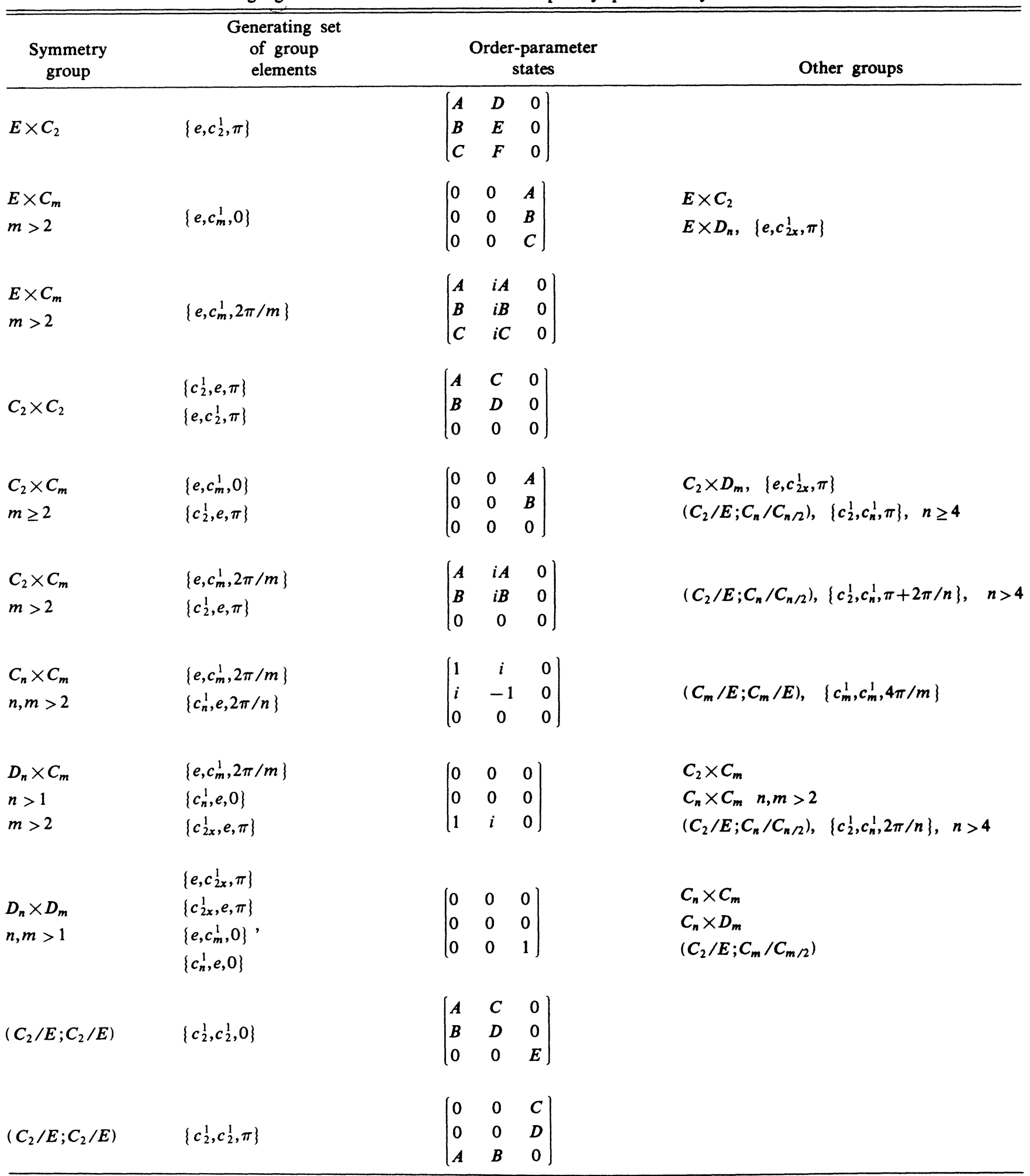


TABLE I. (Continued).

\begin{tabular}{|c|c|c|c|c|c|}
\hline $\begin{array}{l}\text { Symmetry } \\
\text { group }\end{array}$ & $\begin{array}{l}\text { Generating set } \\
\text { of group } \\
\text { elements }\end{array}$ & & $\begin{array}{l}\text { er-parame } \\
\text { states }\end{array}$ & & Other groups \\
\hline$\left(C_{3} / E ; C_{3} / E\right)$ & $\left\{c_{3}^{1}, c_{3}^{1}, 2 \pi / 3\right\}$ & $\mid \begin{array}{c}-A \\
i A \\
C\end{array}$ & $\begin{array}{cc}i A & B \\
A & i B \\
i C & 0\end{array}$ & B & \\
\hline$\left(C_{2} / E ; C_{4} / C_{2}\right)$ & $\left\{c_{2}^{1}, c_{4}^{1},-\pi / 2\right\}$ & $\mid \begin{array}{l}A \\
B \\
C\end{array}$ & $\left.\begin{array}{cc}i A & 0 \\
i B & 0 \\
-i C & 0\end{array}\right]$ & & \\
\hline$\left(C_{4} / E ; C_{4} / E\right)$ & $\left\{c_{4}^{1}, c_{4}^{1}, \pi\right\}$ & $\mid \begin{array}{l}A \\
B \\
0\end{array}$ & $\left.\begin{array}{cc}B & 0 \\
-A & 0 \\
0 & 0\end{array}\right]$ & & $\begin{array}{l}\left(C_{4} / C_{2} ; C_{4} / C_{2}\right) \\
\left(C_{3} / E ; C_{6} / C_{2}\right), \quad\left\{c_{3}^{1}, c_{6}^{1}, \pi\right\}\end{array}$ \\
\hline $\begin{array}{l}\left(C_{m} / E ; C_{m} / E\right) \\
m \geq 3\end{array}$ & $\left\{c_{m}^{1}, c_{m}^{1}, 0\right\}$ & $\mid \begin{array}{c}A \\
-B \\
0\end{array}$ & $\begin{array}{ll}B & 0 \\
A & 0 \\
0 & C\end{array} \mid$ & & \\
\hline $\begin{array}{l}\left(C_{m} / E ; C_{m} / E\right) \\
m \geq 4\end{array}$ & $\left\{c_{m}^{1}, c_{m}^{1}, 2 \pi / m\right\}$ & $\mid \begin{array}{ll}0 & \\
0 & \\
B & i\end{array}$ & $\left.\begin{array}{cc}0 & A \\
0 & i A \\
B B & 0\end{array}\right)$ & & \\
\hline $\begin{array}{l}\left(C_{n} / E ; C_{2 n} / C_{2}\right) \\
n>3\end{array}$ & $\left\{c_{n}^{1}, c_{2 n}^{1}, \pi / n\right\}$ & $\mid \begin{array}{c}A \\
i A \\
B\end{array}$ & $\begin{array}{cc}i A & 0 \\
-A & 0 \\
i B & 0\end{array}$ & & \\
\hline$\left(D_{2} / E ; D_{2} / E\right)$ & $\begin{array}{l}\left\{c_{2}^{1}, c_{2}^{1}, 0\right\} \\
\left\{c_{2 x}^{1}, c_{2 x}^{1}, 0\right\}\end{array}$ & $\mid \begin{array}{ll}A & C \\
0 & 1 \\
0 & C\end{array}$ & $\left.\begin{array}{ll}0 & 0 \\
B & 0 \\
0 & C\end{array}\right)$ & & \\
\hline$\left(D_{2} / E ; D_{2} / E\right)$ & $\begin{array}{l}\left\{c_{2 x}^{1}, c_{2 x}^{1}, \pi\right\} \\
\left\{c_{2}^{1}, c_{2}^{1}, 0\right\}\end{array}$ & $\mid \begin{array}{ll}0 & \\
B & C \\
0 & C\end{array}$ & $\left.\begin{array}{ll}A & 0 \\
0 & 0 \\
0 & 0\end{array}\right)$ & & \\
\hline$\left(D_{4} / C_{2} ; D_{4} / C_{2}\right)$ & $\begin{array}{l}\left\{c_{2, x+y}^{1}, c_{4}^{1}, \pi / 2\right\} \\
\left\{c_{2 x}^{1}, c_{2 y}^{1}, \pi\right\}\end{array}$ & $\mid \begin{array}{ll}1 & 0 \\
0 & i \\
0 & 0\end{array}$ & $\begin{array}{l}0 \\
0 \\
0\end{array} \mid$ & & \\
\hline $\begin{array}{l}\left(D_{m} / E ; D_{m} / E\right) \\
m>2\end{array}$ & $\begin{array}{l}\left\{c_{2 x}^{1}, c_{2 x}^{1}, 0\right\} \\
\left\{c_{m}^{1}, c_{m}^{1}, 0\right\}\end{array}$ & $\mid \begin{array}{ll}A & \\
0 & \\
0 & \end{array}$ & $\left.\begin{array}{ll}0 & 0 \\
A & 0 \\
0 & B\end{array}\right)$ & & \\
\hline $\begin{array}{l}\left(D_{m} / E ; D_{m} / E\right) \\
m>2\end{array}$ & $\left\{c_{2 x}^{1}, c_{2 x}^{1}, \pi\right\}$ & {$\left[\begin{array}{ll}0 & 1 \\
1 & 0 \\
0 & 0\end{array}\right.$} & $\left.\begin{array}{ll}1 & 0 \\
0 & 0 \\
0 & 0\end{array}\right)$ & & \\
\hline$(T / E ; T / E)$ & $\left\{c_{3, x+y+z}^{1}, c_{3, x+y+z}^{1}, 2 \pi / 3\right\}$ & $\mid \begin{array}{ll}1 & \\
0 & e \\
0 & \end{array}$ & $\begin{array}{c}0 \\
e^{-2 \pi i / 3} \\
0\end{array}$ & $\left.\begin{array}{c}0 \\
0 \\
e^{-4 \pi t / 3}\end{array}\right)$ & \\
\hline$(Y / E ; Y / E)$ & $\left\{c_{3, x+y+z}^{1}, c_{3, x+y+z}^{1}, 0\right\}$ & $\left(\begin{array}{ll}1 & 0 \\
0 & 1 \\
0 & 0\end{array}\right.$ & $\left.\begin{array}{ll}0 & 0 \\
1 & 0 \\
0 & 1\end{array}\right)$ & & $\begin{array}{l}(T / E ; T / E) \\
(O / E ; O / E)\end{array}$ \\
\hline
\end{tabular}




$$
\left[\begin{array}{lll}
0 & 0 & A \\
0 & 0 & B \\
C & D & 0
\end{array}\right),
$$

and this state is invariant under $\left(C_{2} / E ; C_{2} / E\right)$;

(iii) another noninert phase found by Jones ${ }^{15}$ is invariant under $C_{2} \times C_{2}$.

This proves that all phases previously found to be stationary points of the free energy contain a residual symmetry given by a subgroup of $G$ (2.4). All other orderparameter structures given in Table I are potential candidates for new stationary points of the free energy. If we subject them to the condition $\partial F / \partial A_{\mu j}=0$, the free, complex parameters in Table I become functions of the coefficients in the free energy. All cases which we were able to investigate in this way lead to phases already known.

\section{SYMMETRY AND STATIONARY POINTS OF THE FREE ENERGY}

The symmetry classification described in Secs. IV and $\mathrm{V}$ does not provide any explicit information about whether the resulting states are stationary points of the free energy or not. On the other hand, the symmetry classification led, for example, to inert phases which are known to be stationary points of the Ginzburg-Landau free energy. ${ }^{13,15}$ Hence there seems to exist a direct connection between the symmetry of a state and the fact that its free energy is a minimum. One may therefore ask the question whether it is possible to predict the existence of stationary states once the underlying symmetry group of the free-energy functional is given. By using methods borrowed from equivariant topology,,$^{42,16}$ it will be shown that the answer is in the affirmative.

To do this, two theorems will be needed whose proof has been given by Michel. ${ }^{42}$ In order to understand these theorems and their bearing on the Ginzburg-Landau theory of ${ }^{3} \mathrm{He}$, a few mathematical terms have to be introduced. For further mathematical details the reader is referred to Refs. 20, 42, and 16.

Mathematically the minimization of the GinzburgLandau functional involves the following ingredients:

(i) a manifold $M$ of complex $3 \times 3$ matrices $A_{\mu j}$ with $\operatorname{Tr}\left(\mathbf{A} \mathbf{A}^{\dagger}\right)=1$

(ii) a group $G$ (2.4) of transformations acting on the manifold;

(iii) a smooth real function $F$ (the free energy) on the manifold, which is invariant under the action of $G$, i.e., for $m \in M$ and arbitrary $g \in G$ one has $F(g m)=F(m)$.

We define the orbit $G(m)$ of a point $m$ by $\boldsymbol{G}(m)=\{\mathrm{gm} \mid g \in G\}$, i.e., $\boldsymbol{G}(\mathrm{m})$ is the set of all points obtained by letting all transformations in $G$ act on $m$. The invariant function $F$ is seen to be constant for all points of an orbit. If $F$ is stationary in a point $m_{0}$, i.e., $\partial F /\left.\partial A_{\mu j}\right|_{m_{0}}=0$, it will be stationary in all points of $G\left(m_{0}\right)$. Hence $G\left(m_{0}\right)$ is a stationary orbit.

Furthermore, the little group $G_{m}$ of a point $m$ is defined as the subgroup of $G$ leaving $m$ invariant, $G_{m}=\{g \in G \mid g m=m\}$ (note that in our case "points of $M$ ' are matrices).
If $m$ and $n$ lie on the same orbit, i.e., if $n=g m$ for some $g \in G$, it follows that $G_{n}=g G_{m} g^{-1}$. In this case the little groups of $n$ and $m$ are conjugated.

All points on the orbit of $m$ belong to little groups conjugated to $G_{m}$. There may be points on other orbits having little groups conjugated to $G_{m}$ as well. This fact leads to the definition of the stratum $S(m)$ of $m$. It is the union of all orbits of points having little groups that are conjugated to $G_{m}$, i.e., $n$ is an element of $S(m)$ if there is an element $g$ in $G$ such that $G_{n}=g G_{m} g^{-1}$.

The stratum is a somewhat abstract notion. One may say that a stratum is the union of all orbits of the same symmetry type: All points of a stratum have conjugated and therefore isomorphic little groups. This may be illustrated in a simple example: As manifold we take $M=S^{2}$, i.e., the sphere in $R^{3}$, and choose $G=\mathrm{SO}(2)=\mathrm{U}(1)$, the rotations about the $z$ axis, as the symmetry group acting on the manifold. The north and south poles of the sphere remain invariant under $G$ : Their respective orbits consist of one point only. The little group of both of them is SO(2). All other points of $M=S^{2}$ are transported along orbits of constant latitude, i.e., circles. These points are invariant under the identity element of $G$ only, hence their little group is $G_{m}=E$. The manifold $M=S^{2}$ can be divided in two strata. One consists of the north and south pole, the other one of the rest of $S^{2}$.

The first important result of $\mathrm{Michel}^{42,16}$ is the following. If an orbit $G(m)$ lies isolated in its stratum $S(m)$, i.e., if there exists a neighborhood $U$ of $G(m)$ such that $U \cap S(m)=\boldsymbol{G}(m)$, then $\boldsymbol{G}(m)$ is a stationary orbit of every smooth real function on $M$ that is invariant under $G$. We note, that if a stratum is composed of finitely many orbits, each of them is isolated in its stratum. This theorem allows one to draw conclusions about superfluid phases of ${ }^{3} \mathrm{He}$. We first consider the $B$ phase. Its invariance group (little group) is $[\mathrm{SO}(3)]_{\vec{S}}+\overrightarrow{\mathrm{L}}$, which determines the $B$-phase order parameter uniquely. It follows that the stratum of the $B$ phase consists of one orbit only, i.e., the orbit is isolated in its stratum. Therefore Michel's theorem states that the $B$ phase is a stationary point of every smooth real function that is invariant under $G(2.4)$. This conclusion is independent of the form of the freeenergy functional. It is not limited to a Ginzburg-Landau type expression valid only close to $T_{c}$. Note, that Balian and Werthamer ${ }^{9}$ showed that within a weak-coupling theory the $B$ phase is indeed the stable state at all temperatures.

The above argument is not only valid for the $B$ phase, but for all inert phases. They can be found explicitly by group theory, as shown in Secs. IV and V. Hence the inert states are stationary points of any smooth free-energy function invariant under $G$ (2.4). Michel's theorem thus makes remarkably general assertions about stationary points without evaluating a single derivative.

The above result had already been conjectured by Jones: ${ }^{15} \mathrm{He}$ tried to construct all algebraically independent polynomials in $A_{\mu j}$ invariant under $G$ (2.4). If all such polynomials were known, it would be sufficient to prove that the inert states are stationary points of these polynomials. However, so far it was not possible to prove their independence and completeness. The geometrical 
method used here avoids these problems altogether.

Michel's theorem cannot be applied to the noninert phases: as their structure depends on the coefficients in the free-energy functional, they elude a theorem making a statement about all $G$-invariant functions. Nonetheless, there are special cases where it is possible to use a second theorem by Michel. ${ }^{42}$ For our purposes it can be formulated as follows.

Let $m$ be a point of the manifold $M$. If the little group $G_{m}$ is maximal in the set of little groups (i.e., if there is no other little group containing $G_{m}$ ) then each $G$ invariant smooth real function $F$ on $M$ is stationary in at least two orbits of $S(m)$. However, now the stationary orbits depend on the function $F$.

We note that there can be several maximal little groups, because two little groups may not be comparable at all (neither $G_{n} \subset G_{m}$ nor $G_{m} \subset G_{n}$ ).

This theorem does not lead to new results for $G=\mathbf{S O}(3) \times \mathbf{S O}(3) \times \mathbf{U}(1)$ : The maximal little groups like $[\mathrm{SO}(3)]_{\overrightarrow{\mathrm{S}}+\overrightarrow{\mathrm{L}}}$ produce inert phases. Hence Michel's first theorem can be applied, which is much stronger than the second one. If, however, the symmetry group is restricted by an external magnetic field or by taking into account coupling of nuclear dipole moments as discussed in Sec. VII, the second theorem can be useful.

\section{SYMMETRY REDUCTION DUE TO A MAGNETIC FIELD OR SPIN-ORBIT COUPLING}

An external magnetic field defines a direction and hence breaks the symmetry with respect to rotations in spin space. The symmetry group of superfluid ${ }^{3} \mathrm{He}$ in a magnetic field $G_{\text {mag }}$ is therefore smaller than $G(2.4)$, i.e., $G_{\text {mag }} \subset G$. The order parameter couples to the magnetic field via the terms ${ }^{11} H_{\mu}\left(\mathrm{AA}^{\dagger}\right)_{\mu v} H_{v}$ and $i \epsilon_{\mu \nu \lambda} H_{\mu}\left(\mathrm{AA}^{\dagger}\right)_{\nu \lambda}$. Therefore in spin space only rotations about the magnetic field leave the free energy invariant. The symmetry-group $G_{\text {mag }}$ then takes the form ${ }^{41}$

$$
G_{\mathrm{mag}}=[\mathrm{U}(1)]_{S_{z}} \times[\mathrm{SO}(3)]_{\overrightarrow{\mathrm{L}}} \times[\mathrm{U}(1)]_{\phi} .
$$

If we use the symmetry classification given before and take into account those groups which in spin space contain only rotations about the $z$ axis, we find that the polar, $A, \beta$, and $A_{1}$ phases are produced explicitly by subgroups of $G_{\text {mag }}$. According to Michel's first theorem, these phases are stationary points of all free-energy functions invariant under $G_{\text {mag }}$. As before Michel's second theorem gives no result.

The symmetry group of ${ }^{3} \mathrm{He}$ subject to the dipole interaction is

$$
\begin{aligned}
G_{\mathrm{dip}} & =[\mathrm{SO}(3)]_{\overrightarrow{\mathrm{s}}+\overrightarrow{\mathrm{L}}} \times[\mathrm{U}(1)]_{\phi} \\
& =(\mathrm{SO}(3) / E ; \mathrm{SO}(3) / E) \times \mathrm{U}(1) .
\end{aligned}
$$

In the presence of dipolar coupling the spin and angular momentum projections $S_{\mathbf{z}}$ and $L_{\mathbf{z}}$ cease to be good quantum numbers: nevertheless, the $3 \times 3$ matrix $A_{\mu j}$ may be taken as an order parameter. ${ }^{33}$ The pair interaction dominates the dipolar interaction by six orders of magnitude, so that we may assume it to determine the overall structure of the order parameter. The dipolar interaction, as a small perturbation, then chooses a state from a manifold of degenerate ones.

The symmetry classification of (7.2) can easily be found from the results obtained in Secs. IV and V. We have to take those subgroups in which operations in spin space are compensated by identical operations in orbit space. We find that the $A_{1}$, planar, $\alpha$, and $B$ phases are given explicitly by symmetry requirements. From this we can conclude that these phases are stationary points of all freeenergy functions invariant under $G_{\text {dip }}$.

In order to find other phases by Michel's second theorm, we have to know all maximum little subgroups of $G_{\text {dip }}$. We find that $[\mathrm{U}(1)]_{S_{z}+L_{z}-\phi}=((\mathrm{U}(1) ; \mathrm{U}(1)) ; \mathrm{U}(1))$ is a maximal little group that does not lead to an inert phase, but to

$$
\left(\begin{array}{ccc}
0 & 0 & A \\
0 & 0 & i A \\
B & i B & 0
\end{array}\right) .
$$

Michel's second theorem allows one to conclude that among all matrices of this form there are at least two stationary points for any free energy.

If we insert (7.3) in (2.5) and require $\partial F / \partial A_{\mu j}=0$, one finds that $A$ and $B$ in (7.3) can be chosen such that this equation has indeed two solutions.

Finally, a combination of the symmetry restrictions due to a magnetic field and the dipolar coupling leads to a symmetry group

$$
\begin{aligned}
G_{m, \text { dip }} & =[\mathrm{U}(1)]_{S_{\mathrm{z}}+L_{\mathrm{z}}} \times[\mathrm{U}(1)]_{\phi} \\
& =(\mathrm{U}(1) / E ; \mathrm{U}(1) / E) \times \mathrm{U}(1) .
\end{aligned}
$$

The subgroups are easy to find. One example is $[\mathrm{U}(1)]_{S_{z}+\phi} \times[\mathrm{U}(1)]_{L_{z}-\phi}$, leading to the $A_{1}$ phase. Hence the $A_{1}$ phase is a stationary state of the most general free-energy function which is invariant under $G_{m \text {,dip. }}$ Indeed the phase diagram of ${ }^{3} \mathrm{He}$ shows a small wedge of the $A_{1}$ phase just below $T_{c}$.

There are two nontrivial maximal little subgroups of $G_{m, \text { dip }}:[\mathrm{U}(1)]_{S_{z}+L_{z}}=(\mathrm{U}(1) ; \mathrm{U}(1))$, leading to the oblate phase (4.20), and again [U(1) $]_{S_{z}+L_{z}-\phi}$, i.e., the $\epsilon$ phase. In this case Michel's second theroem can be applied and predicts at least two stationary points in the strata of the oblate and the $\epsilon$ phase. It is satisfactory to see the oblate phase appear at this point: the $B_{2}$ phase (4.20), i.e., the $B$ phase in a magnetic field, lies in the stratum of the oblate phase. If we consider the $B$ phase in the presence of dipolar coupling and neglect the magnetic field for a moment, we are led to the oblate phase as well: the dipolar interaction favors a state in which spin and orbit space are rotated against each other by ${ }^{33} \gamma=104^{\circ}$. If we choose the $z$ axis of spin space to be the rotation axis, the order parameter of the $B$ phase is transformed to the oblate form. Hence the structure of the $B_{2}$ phase is determined by symmetry. 


\section{SYMMETRY CLASSIFICATION OF EXACTLY SOLVABLE MODELS}

As shown in Secs. IV-VI the symmetry classification of $G$ (2.4) yields all order-parameter states found previously by minimization of the Ginzburg-Landau functional (2.5). This supports the observation discussed in Sec. I that minima of the free energy usually correspond to states with a remaining internal symmetry.

It is worthwhile to verify this point explicitly in the case of other systems described by a tensor order parameter, whose free-energy functional is nonetheless simpler than (2.5). Two examples will be presented.

(1) If, hypothetically, the feature of superfluidity of ${ }^{3} \mathrm{He}$-corresponding to the breaking of the U(1) symmetry in (2.4) - did not exist, while the other properties were still present, the order-parameter $A_{\mu j}$ would be a real matrix. The underlying symmetry of the free-energy functional would then be given by

$$
G=[\mathrm{SO}(3)]_{\overrightarrow{\mathrm{S}}} \times[\mathrm{SO}(3)]_{\overrightarrow{\mathrm{L}}} .
$$

Because of $\mathbf{A}=\mathbf{A}^{*}$ the free energy in the GinzburgLandau regime can be obtained from (2.5) by putting $\beta_{1}=\beta_{2} \equiv \gamma_{1} / 2, \beta_{3}=\beta_{4}=\beta_{5} \equiv \gamma_{2} / 3$, i.e.,

$F=\alpha \Delta^{2} \operatorname{Tr}(\mathbf{A} \tilde{\mathbf{A}})+\frac{1}{2} \Delta^{4}\left\{\gamma_{1}[\operatorname{Tr}(\mathbf{A} \tilde{\mathbf{A}})]^{2}+\gamma_{2}\left[\operatorname{Tr}(\mathbf{A} \tilde{\mathbf{A}})^{2}\right]\right\}$.

To minimize (8.2) it is convenient to represent the $3 \times 3$ matrix $A_{\mu j}$ by three vectors (i.e., a triad), and their relative orientations in terms of three angles. One finds that there are exactly two minima.

(i) For $\gamma_{1}+\gamma_{2}>0$ and $\gamma_{2}>0$ the minimum is given by an order parameter where the three vectors lie mutually perpendicular and have the same length. This is the order parameter of the BW state; the corresponding energy is given by $F=-\frac{3}{2} \alpha^{2} /\left(3 \gamma_{1}+\gamma_{2}\right)$.

(ii) For $\gamma_{1}+\gamma_{2}>0$ and $\gamma_{1}<0$ the order parameter of the minimum can be represented by an (anti) parallel orientation of all three vectors where only the sum of the square of their lengths is determined by (8.2). This corresponds to the polar state. The energy is given $F=-\frac{1}{2} \alpha^{2} /\left(\gamma_{1}+\gamma_{2}\right)$.

Next, we apply the concept of symmetry classification to (8.1). The result easily follows from those obtained in Secs. IV and V by neglecting the phase [i.e., U(1)] degree of freedom. Concerning the continuous symmetries this implies putting $c=0$ in (3.11). Therefore, the states (1)-(5) in Sec. IV (with real matrix elements) are possible candidates for phases with a remaining symmetry: the BW state with a $\mathrm{SO}(3)$ symmetry, the polar state with a $\mathrm{U}(1) \times \mathrm{U}(1)$ symmetry, and three more states with a remaining $U(1)$ symmetry. The comparison with the analytic solution shows that the minima of (8.2) are indeed the states with the highest remaining symmetry.

(2) Even more interesting are the states thought to describe the bulk matter of neutron stars. ${ }^{43}$ They are assumed to be due to Cooper pairing of neutrons into a ${ }^{3} P_{2}$ state ( $l=1, S=1$, and $J=2$ because of strong spin-orbit coupling). In order to describe a $J=2$ state the order parameter has to be a traceless symmetric tensor $B_{\mu v}$. (This also holds for $l=2$ pairing ${ }^{11}$ in superfluid ${ }^{3} \mathrm{He}$. Indeed, as shown by Sauls and Serene, ${ }^{44}$ the Ginzburg-Landau functionals for the two problems are identical. Hence the results of Mermin ${ }^{45}$ for the stable states in the case of $l=2$ pairing may be used.) In the case of such a pairing the free energy has to be invairant under joint threedimensional rotations in spin and orbit space and a gauge transformation, ${ }^{45,46}$ i.e.,

$$
G=[\mathrm{SO}(3)]_{\vec{J}} \times[\mathrm{U}(1)]_{\phi} .
$$

In the Ginzburg-Landau regime the free energy compatible with this symmetry can be obtained from (2.5) by putting ( $\mathbf{A} \equiv) \mathbf{B}=\widetilde{\mathbf{B}}$ and using the appropriate Fierz identity for traceless $3 \times 3$ tensors. One finds ${ }^{11,46}$

$$
\begin{aligned}
& F=\alpha \Delta^{2} \operatorname{Tr}\left(\mathbf{B B}^{*}\right) \\
&+\frac{1}{2} \Delta^{4}\left\{\beta_{1}\left|\operatorname{Tr} \mathbf{B}^{2}\right|^{2}+\beta_{2}\left[\operatorname{Tr}\left(\mathbf{B B}^{*}\right)\right]^{2}\right. \\
&\left.+\beta_{3} \operatorname{Tr}\left(\mathbf{B}^{2} \mathbf{B}^{* 2}\right)\right\},
\end{aligned}
$$

where we again assume $\mathbf{B}$ to be normalized $\left[\operatorname{Tr}\left(\mathbf{B B}^{*}\right)=1\right]$.

This functional has three fourth-order invariants. The minimization of (8.4) is quite nontrivial but can still be performed analytically as shown by Mermin. ${ }^{46}$ Depending on the $\beta_{i}$ there are three distinct absolute minima.

(i) For $\beta_{3}>-\beta_{1}+\left|\beta_{1}\right|$ the order parameter is given by

$$
B_{\mu v}=\frac{1}{2}\left(\begin{array}{ccc}
1 & i & 0 \\
i & -1 & 0 \\
0 & 0 & 0
\end{array}\right),
$$

which has the form of the $A_{1}$ phase (4.36) and whose energy is $F=-\alpha^{2} / 2 \beta_{2}$.

(ii) For $-6 \beta_{1}<\beta_{3}<0$ the matrix has the form

$$
B_{\mu \nu}=\left(\begin{array}{ccc}
1 & 0 & 0 \\
0 & e^{2 \pi i / 3} & 0 \\
0 & 0 & e^{4 \pi i / 3}
\end{array}\right),
$$

which is the $\alpha$ phase; its free energy is given by $F=-\alpha^{2} /\left[2\left(\beta_{2}+\beta_{3} / 3\right)\right]$.

(iii) For $\beta_{3}<-4 \beta_{1}-2\left|\beta_{1}\right|$ the (highly degenerate) minimum is given by any real matrix $B_{\mu}$ (up to a phase factor); its energy is found as $F=-\alpha^{2}$ / $\left[2\left(\beta_{1}+\beta_{2}+\beta_{3} / 2\right)\right]$.

Now to the symmetry classification of (8.3). This is again easily done by noting that the symmetry (8.3) corresponds to that of superfluid ${ }^{3} \mathrm{He}$ with a dipolar coupling. Its symmetry classification has been discussed in Sec. VII. There are three continuous subgroups: $[\mathrm{U}(1)]_{J_{z}-\phi}$, $[\mathrm{U}(1)]_{2 J_{z}-\phi}$, and $[\mathrm{U}(1)]_{J_{z}}$. The corresponding order parameters are found from (4.6) by setting $a_{z}=b_{z}=c / 2$, $a_{z}=b_{z}=c$, and $a_{z}=b_{z}, c=0$, respectively. All three yield inert states. In the first case, $B$ has the form (8.5) which is the first of the global minima found by Mermin. ${ }^{46}$ In the second case, $B$ is obtained as (4.40), the $\epsilon$ phase, with $A=B=1$; although a stationary point, it does not correspond to a global minimum. In the third case, $B$ is given by a real, traceless matrix with an overall phase factor, corresponding to the third stable phase found by Mermin. ${ }^{46}$ The discrete symmetries yield two more inert 
phases: $(T / E ; T / E)$ leads to the $\alpha$ phase (8.6), i.e., the second minimum of the free energy, and $\left(D_{m} / E ; D_{m} / E\right)$ to the analogue of the planar phase, which, however, is always a saddle point of the free energy. There are some more discrete symmetries (more restrictive than the two mentioned above) which lead to noninert states.

Therefore we find that all minima of (8.5) are included in a symmetry classification, i.e., can be obtained by symmetry alone. Here we were able to verify explicitly the importance of discrete symmetries.

\section{CONCLUSION}

Using group-theoretical methods a complete classification of the continuous and discrete subgroups of the symmetry group $G=[\mathrm{SO}(3)]_{\overrightarrow{\mathrm{S}}} \times[\mathrm{SO}(3)]_{\overrightarrow{\mathrm{L}}} \times[\mathrm{U}(1)]_{\phi}$, relevant for superfluid ${ }^{3} \mathrm{He}$, has been presented. The states invariant under their action have been explicitly determined. We have shown that all phases previously found in the literature (i.e., by minimizing the Ginzburg-Landau free energy) are included in this classification. The inert states can be obtained directly from symmetry requirements, there are no free parameters to be adjusted. The noninert states are not given by symmetry alone; however, they appear naturally in the classification, because each of them is also invariant under a group of transformations. Several states of a new symmetry type were derived. The concept of broken relative symmetry appears as a natural feature in such a group-theoretical treatment.

The invariance under a group of transformations does not generally prove a state to be a stationary point of the free energy. The investigation of this question in the second part of our work led us to the conclusion that the eight inert phases are stationary points of any free energy invariant under the symmetry-group $G=\mathrm{SO}(3)$ $\times \mathrm{SO}(3) \times \mathrm{U}(1)$. In particular, this conclusion is independent of any Ginzburg-Landau expansion which is only valid in the vicinity of the phase transition.

Concerning the noninert states only less strict results hold: It was shown that some of them are stationary points of an arbitrary free-energy functional if their parameters are adjusted properly. It is remarkable, however, that both results are based on purely geometrical arguments: It was not necessary to calculate any derivatives.

These results were also applied to ${ }^{3} \mathrm{He}$ in a magnetic field and ${ }^{3} \mathrm{He}$ subjected to dipolar coupling, respectively. In both cases the symmetry classification again yielded all the phases obtained by physical reasoning or exact minimization. Applying the classification to two models of order parameters whose free-energy functional is slightly simpler than that for ${ }^{3} \mathrm{He}$ (e.g., those describing $d$-wave pairing in superfluid ${ }^{3} \mathrm{He}$ or superfluidity in neutron stars) and which can be minimized analytically, we have explicitly proven that a symmetry classification yields all minima.

We conclude that group-theoretical methods employing symmetry considerations are a very valuable tool for the investigation of the stationary points of free-energy functionals.

\section{ACKNOWLEDGMENTS}

We are particularly grateful to Peter Wölfle for many useful discussions. One of us (D.V.) would like to thank Mario Liu for valuable comments, and Heinrich Saller and Ulli Wolff for helpful discussions on group theory. One of us (D.V.) acknowledges support from the Deutsche Forschungsgemeinschaft (Bonn, Germany).
"Present address: Institut für Theoretische Physik, Eidgenössische Technische Hochschule Zürich, CH-8093 Zürich, Switzerland.

${ }^{1}$ A. J. Leggett, Rev. Mod. Phys. 47, 331 (1975).

${ }^{2}$ P. W. Anderson and W. F. Brinkmann, in The Physics of Liquid and Solid Helium, Part II, edited by K. H. Bennemann and J. B. Ketterson (Wiley, New York, 1978).

${ }^{3}$ P. Wölfle, Rep. Prog. Phys. 42, 269 (1979).

${ }^{4} \mathrm{P}$. Wölfle and D. Vollhardt, The Superfluid Phases of ${ }^{3} \mathrm{He}$ (Francis and Taylor, London, in press).

5J. C. Wheatley, Rev. Mod. Phys. 47, 415 (1975).

${ }^{6}$ D. M. Lee and R. C. Richardson, see Ref. 2.

${ }^{7}$ L. D. Landau and E. M. Lifshitz, Statistical Physics (Pergamon, London, 1958).

${ }^{8}$ C. Itzykson and J.-B. Zuber, Quantum Field Theory (McGraw-Hill, New York, 1980).

${ }^{9}$ R. Balian and N. R. Werthamer, Phys. Rev. 131, 1553 (1963).

${ }^{10}$ V. Ambegaokar and N. D. Mermin, Phys. Rev. Lett. 30, 81 (1973).

${ }^{11}$ N. D. Mermin and C. Stare, Phys. Rev. Lett. 30, 1135 (1973).

${ }^{12}$ N. D. Mermin and C. Stare, Cornell University Report No. 2186, 1974 (unpublished).

${ }^{13}$ G. Barton and M. A. Moore, J. Phys. C 7, 4220 (1974).

${ }^{14} \mathrm{G}$. Barton and M. A. Moore, J. Phys. C 8, 970 (1975).

${ }^{15}$ R. B. Jones, J. Phys. C 10, 657 (1977).
${ }^{16}$ L. Michel, Rev. Mod. Phys. 52, 617 (1980).

${ }^{17}$ M. V. Jarić, in Group Theoretical Methods in Physics, proceedings of the IX International Colloquium, Cocoyoc, Mexico, 1980, edited by K. B. Wolf (Lecture Notes in Physics, Vol. 135) (Springer-Verlag, Berlin, 1980).

18J. S. Kim, Nucl. Phys. B196, 285 (1982).

${ }^{19}$ S. Frautschi and J. S. Kim, Nucl. Phys. B196, 301 (1982).

${ }^{20}$ M. Abud and G. Sartori, Ann. Phys. (N.Y.) 150, 307 (1983).

${ }^{21}$ M. V. Jarić, Phys. Rev. Lett. 48, 1641 (1982).

22J. S. Kim, Phys. Rev. B 31, 1433 (1985).

${ }^{23}$ V. L. Golo and M. I. Monastyrskiy, Pis'ma Zh. Eksp. Teor. Fiz. 25, 272 (1977) [Sov. Phys.-JETP Lett. 25, 251 (1977)]; Institute of Theoretical and Experimental Physics, Moscow ITEP-173, 1976 (unpublished).

${ }^{24}$ V. P. Mineev, Sov. Sci. Rev. Sect. A 2, 173 (1980).

25P. W. Anderson, Phys. Rev. B 30, 4000 (1985).

${ }^{26}$ G. E. Volovik and L. P. Gorkov, Zh. Eksp. Teor. Fiz. 88, 1412 (1985) [Sov. Phys._JETP 61, 843 (1985)].

${ }^{27}$ K. Ueda and T. M. Rice, Phys. Rev. B 31, 7114 (1985).

${ }^{28}$ E. I. Blount, Phys. Rev. B 32, 2935 (1985).

${ }^{29}$ M. M. Salomaa and G. E. Volovik, Phys. Rev. B 31, 203 (1985).

${ }^{30}$ P. W. Anderson and W. F. Brinkman, Phys. Rev. Lett. 30, 1108 (1973).

${ }^{31}$ D. Rainer and J. W. Serene, Phys. Rev. B 13, 4745 (1976). 
32J. Sauls and J. W. Serene, Physica 108B +C, 1137 (1981).

${ }^{33}$ A. J. Leggett, J. Phys. C 6, 3187 (1973).

${ }^{34}$ M. Liu and M. C. Cross, Phys. Rev. Lett. 41, 250 (1978).

${ }^{35}$ M. Liu, Physica 109B + C, 1615 (1982).

${ }^{36}$ M. Liu and M. C. Cross, Phys. Rev. Lett. 43, 296 (1979).

${ }^{37}$ M. Liu, Phys. Rev. Lett. 43, 1740 (1979).

${ }^{38}$ L. D. Landau and E. M. Lifshitz, Quantum Mechanics (Pergamon, London, 1958).

39J. C. Toledano, L. Michel, P. Toledano, and E. Brezin, Phys. Rev. B 31, 7171 (1985).

${ }^{40} \mathrm{C}$. Bruder, Diploma thesis, Technical University of Munich,
1985 (unpublished)

${ }^{41}$ G. E. Volovik and V. P. Mineev, Zh. Eksp. Teor. Fiz. 72, 2256 (1977) [Sov. Phys.-JETP 45, 1186 (1977)].

${ }^{42}$ L. Michel, C. R. Acad. Sci. (Paris) A272, 433 (1971).

${ }^{43}$ See, for example, D. Pines and A. Alpar, Nature (London) 316, 27 (1985)

44J. A. Sauls and J. W. Serene, Phys. Rev. D 17, 1524 (1978).

${ }^{45}$ N. D. Mermin, in Quantum Liquids, edited by J. Ruvalds and T. Regge (North-Holland, Amsterdam, 1978), p. 195.

46N. D. Mermin, Phys. Rev. A 9, 868 (1974) 